\title{
Experimental and DFT Studies on the Identity Exchange Reactions Between Phenyl Chalcogen Iranium Ions and Alkenes
}

\section{SUPPORTING INFORMATION}

Samuel C. Brydon ${ }^{\mathrm{a}}$, Zhonghua Ren ${ }^{\mathrm{b}}$, Gabriel da Silva ${ }^{\mathrm{b}}$, S. Fern Lim ${ }^{\mathrm{a}}$, George N. Khairallah ${ }^{\mathrm{a}, \mathrm{c}}$, Michael J. Rathjen ${ }^{\mathrm{a}}$, Jonathan M. White ${ }^{\mathrm{a}^{*}}$, Richard A. J. O'Hair ${ }^{\mathrm{a}^{*}}$

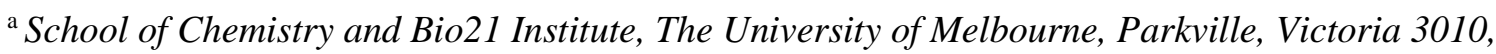
Australia.

${ }^{b}$ Chemical and Biomolecular Engineering, The University of Melbourne, Parkville, Victoria 3010, Australia.

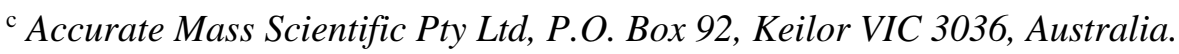

*Corresponding authors' emails: rohair@unimelb.edu.au; whitejm@unimelb.edu.au 
Experimental: Synthesis of deuterated chalcogenyl ethanol substrates 1a-3a.

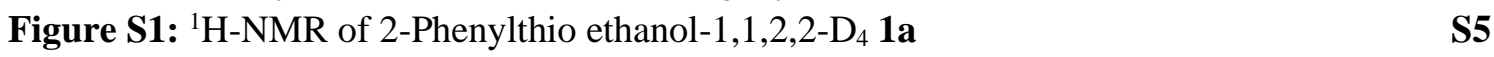

Figure S2: ${ }^{13} \mathrm{C}-\mathrm{NMR}$ of 2-Phenylthio ethanol-1,1,2,2- $\mathrm{D}_{4} \mathbf{1 a} \quad$ S6

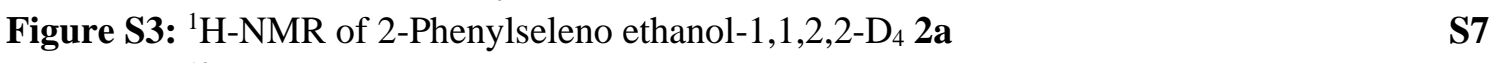

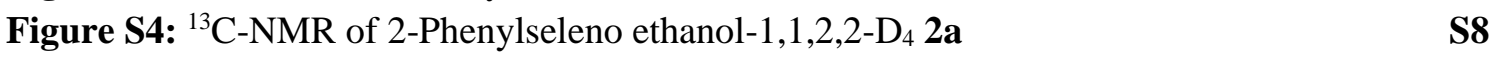

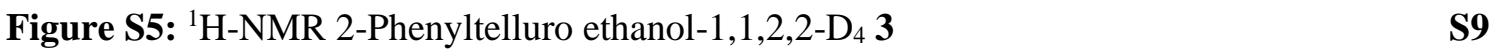

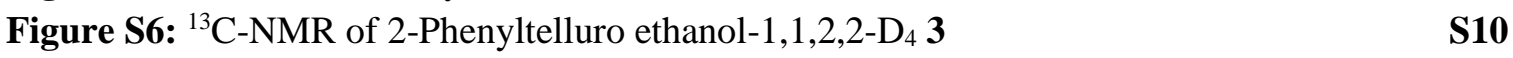

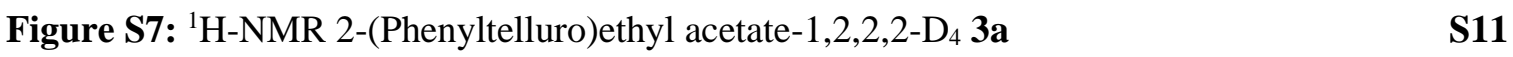

Figure S8: ${ }^{13} \mathrm{C}-\mathrm{NMR}$ of 2-(Phenyltelluro)ethyl acetate-1,2,2,2- $\mathrm{D}_{4}$ 3a $\quad$ S12

Figure S9. Experimental and theoretical high-resolution accurate mass measurements for the ions corresponding to thiiranium ion $\mathbf{1 b}\left[\mathrm{C}_{8} \mathrm{H}_{5} \mathrm{D}_{4} \mathrm{~S}\right]^{+}(\mathrm{m} / z$ 141). $\quad \mathbf{S 1 3}$

Figure S10. Experimental and theoretical high-resolution accurate mass measurements for the ions corresponding to seleniranium ion $2 \mathbf{b}\left[\mathrm{C}_{8} \mathrm{H}_{5} \mathrm{D}_{4} \mathrm{Se}\right]^{+}(\mathrm{m} / z$ 189).

Figure S11. Experimental and theoretical high-resolution accurate mass measurements for the ions corresponding to telluriranium ion $\mathbf{3 b}\left[\mathrm{C}_{8} \mathrm{H}_{5} \mathrm{D}_{4} \mathrm{Te}\right]^{+}(\mathrm{m} / z 2239)$.

Figure S12. Experimental and theoretical high-resolution accurate mass measurements for the ions corresponding to thiiranium ion $4 \mathbf{b}\left[\mathrm{C}_{12} \mathrm{H}_{15} \mathrm{~S}\right]^{+}(\mathrm{m} / z \mathrm{z} 191)$.

Figure S13. Experimental and theoretical high-resolution accurate mass measurements for the ions corresponding to seleniranium ion $\mathbf{5 b}\left[\mathrm{C}_{12} \mathrm{H}_{15} \mathrm{Se}\right]^{+}(\mathrm{m} / z$ 239).

Figure S14. Experimental and theoretical high-resolution accurate mass measurements for the ions corresponding to telluriranium ion $\mathbf{6} \mathbf{b}\left[\mathrm{C}_{12} \mathrm{H}_{15} \mathrm{Te}\right]^{+}(\mathrm{m} / z 289)$.

Figure S15: Early onset of the reaction of telluriranium ion $\mathbf{3 b}\left(\mathrm{m} / z\right.$ 239) with $\mathrm{C}_{2} \mathrm{H}_{4}$

Figure S16: Early onset of the reaction of seleniranium ion $\mathbf{5 b}(\mathrm{m} / z 2239)$ with $c-\mathrm{C}_{6} \mathrm{D}_{10}$

Figure S17: Early onset of the reaction of telluriranium ion $6 \mathbf{b}(\mathrm{m} / z 289)$ with $c-\mathrm{C}_{6} \mathrm{D}_{10}$

Gaussian Cartesian Coordinates and Energies S18- 


\section{Experimental}

Nuclear Magnetic Resonance Spectroscopy: Proton and proton decoupled carbon NMR spectra for ${ }^{1} \mathrm{H}$ and ${ }^{13} \mathrm{C}$ nuclei respectively were recorded at ambient temperature $\left(25^{\circ} \mathrm{C}\right)$ as solutions in the stated solvent using a Varian Inova 400 NMR spectrometer.

Synthesis of 2-Phenylthio ethanol-1,1,2,2- $D_{4}$ 1a

To a solution of thiophenol $(0.500 \mathrm{~mL}, 4.90 \mathrm{mmol})$ in aq. $\mathrm{NaOH}\left(10 \%\right.$ in $\left.\mathrm{H}_{2} \mathrm{O}\right)$ was added dropwise 2bromoethanol-1,1,2,2- $\mathrm{D}_{4}(0.350 \mathrm{~mL}, 4.94 \mathrm{mmol})$, and the mixture refluxed for $1 \mathrm{~h}$ under nitrogen. Once cooled, the reaction mixture was extracted with $\mathrm{CH}_{2} \mathrm{Cl}_{2}(3 \times 10 \mathrm{~mL})$, the combined organic fractions dried $\left(\mathrm{MgSO}_{4}\right)$, and solvent removed in vacuo to give the pure product $(0.759 \mathrm{~g}, 98 \%$, clear oil $)$.

${ }^{1} \mathbf{H}$ NMR $\left(\mathbf{C D C l}_{3}, 400 \mathrm{MHz}\right) \delta 7.39(\mathrm{~d}, J=7.8 \mathrm{~Hz}, 2 \mathrm{H}, \mathrm{Ar}-\boldsymbol{H}), 7.30(\mathrm{t}, J=7.4 \mathrm{~Hz}, 2 \mathrm{H}, \mathrm{Ar}-\boldsymbol{H}), 7.24-$ $7.20(\mathrm{~m}, 1 \mathrm{H}, \mathrm{Ar}-\boldsymbol{H}) 1.93$ (bs, $1 \mathrm{H},-\mathrm{OH}) .{ }^{13} \mathbf{C} \mathbf{~ N M R}\left(\mathbf{C D C l}_{3}, \mathbf{1 0 0} \mathbf{M H z}\right) \delta 134.9,130.0,129.1,126.6$

Synthesis of 2-Phenylseleno ethanol-1,1,2,2- $D_{4} \mathbf{2 a}$

To a solution of diphenyl diselenide $(97.7 \mathrm{mg}, 0.313 \mathrm{mmol})$ in $\mathrm{EtOH}(5 \mathrm{~mL})$ was added sodium borohydride (42.4 mg, $1.12 \mathrm{mmol}$ ), and the mixture stirred at RT with effervescence. To the colourless solution was added 2-bromoethanol-1,1,2,2- $\mathrm{d}_{4}(45.4 \mu \mathrm{L}, 0.641 \mathrm{mmol})$ dropwise, and the mixture heated to $80^{\circ} \mathrm{C}$ and stirred for $4 \mathrm{~h}$ under nitrogen. Once cooled, the residue was treated with $\mathrm{H}_{2} \mathrm{O}(10 \mathrm{~mL})$ and extracted with $\mathrm{CH}_{2} \mathrm{Cl}_{2}(3 \times 20 \mathrm{~mL})$. The combined organic fractions with dried $\left(\mathrm{MgSO}_{4}\right)$, and solvent removed via rotary evaporation to give the crude product. Purification by column chromatography (Silica, 30-100\% EtAc in PS 40-60) gave the product (95.2 mg, 74\%, clear oil).

${ }^{1} \mathbf{H}$ NMR $\left(\mathbf{C D C l}_{3}, \mathbf{4 0 0} \mathbf{M H z}\right) \delta 7.53(\mathrm{dd}, J=3.1,4.7 \mathrm{~Hz}, 2 \mathrm{H}, \mathrm{Ar}-\boldsymbol{H}), 7.27-7.26(\mathrm{~m}, 3 \mathrm{H}, \mathrm{Ar}-\boldsymbol{H}), 2.01$ (bs, $1 \mathrm{H},-\mathrm{OH}) .{ }^{13} \mathbf{C} \mathbf{~ N M R}\left(\mathbf{C D C l}_{\mathbf{3}}, \mathbf{1 0 0} \mathbf{M H z}\right) \delta 133.1,129.2,128.7,127.3$

Synthesis of 2-Phenyltelluro ethanol-1,1,2,2- $D_{4} \mathbf{3}$

To a solution of diphenyl ditelluride $(97.7 \mathrm{mg}, 0.239 \mathrm{mmol})$ in $\mathrm{THF}(6 \mathrm{~mL})$ at $0^{\circ} \mathrm{C}$, was added sodium borohydride (34.3 mg, $0.907 \mathrm{mmol}$ ) and then EtOH dropwise until effervescence occurred. To the clear solution was added 2-bromoethanol-1,1,2,2- $\mathrm{d}_{4}(35.0 \mu \mathrm{L}, 0.490 \mathrm{mmol})$ dropwise at $0^{\circ} \mathrm{C}$, and the mixture left to stir to RT under nitrogen in the dark. After $4 \mathrm{~h}$, solvent was removed via rotary evaporation and the residue diluted with $\mathrm{H}_{2} \mathrm{O}(10 \mathrm{~mL})$ and then extracted with $\mathrm{CH}_{2} \mathrm{Cl}_{2}(3 \times 10 \mathrm{~mL})$. The combined organic fractions were dried $\left(\mathrm{MgSO}_{4}\right)$ and solvent removed in vacuo to give an orange oil. The crude product was purified by column chromatography (Silica, 10-60\% EtAc in PS 40-60) to give the product (98.1 mg, 81\%, yellow oil).

${ }^{1} \mathrm{H}$ NMR $\left(\mathrm{CDCl}_{3}, 400 \mathrm{MHz}\right) \delta 7.75(\mathrm{~d}, J=7.8 \mathrm{~Hz}, 2 \mathrm{H}, \mathrm{Ar}-\boldsymbol{H}), 7.32-7.28(\mathrm{~m}, 1 \mathrm{H}, \mathrm{Ar}-\boldsymbol{H}), 7.23-7.19(\mathrm{~m}$, 2H, Ar-H), $1.90(\mathrm{bs}, 1 \mathrm{H},-\mathrm{OH}) .{ }^{13} \mathbf{C}$ NMR $\left(\mathbf{C D C l}_{3}, \mathbf{1 0 0} \mathbf{M H z}\right) \delta 138.6,129.8,129.3,127.9$ 
Synthesis of 2-(Phenyltelluro)ethyl acetate-1,2,2,2- $D_{4} \mathbf{3 a}$

To a solution of alcohol $\mathbf{3}$ (90.0 $\mathrm{mg}, 0.355 \mathrm{mmol})$ in pyridine $(3 \mathrm{~mL})$, was added acetic anhydride (50 $\mu \mathrm{L})$ and solution left to stir at RT overnight. Solvent was removed via rotary evaporation and the residue purified by column chromatography (Silica, 0-60\% EtAc in PS 40-60) to give the product (60.0 mg, $57 \%$, yellow oil).

${ }^{1} \mathbf{H}$ NMR $\left(\mathbf{C D C l}_{3}, 400 \mathrm{MHz}\right) \delta 7.75(\mathrm{~d}, J=7.4 \mathrm{~Hz}, 2 \mathrm{H}, \mathrm{Ar}-\boldsymbol{H}), 7.28(\mathrm{~d}, J=7.43 \mathrm{~Hz}, 1 \mathrm{H}, \mathrm{Ar}-\boldsymbol{H}), 7.17-$ $7.25(\mathrm{~m}, 2 \mathrm{H}, \mathrm{Ar}-\boldsymbol{H}), 1.99$ (s, 3H, $\left.-\mathrm{C}(=\mathrm{O}) \mathrm{CH}_{3}\right) .{ }^{13} \mathbf{C} \mathbf{~ N M R}\left(\mathbf{C D C l}_{3}, \mathbf{1 0 0} \mathbf{M H z}\right) \delta 170.7,138.6,129.6$, $129.3,128.0,20.9$. 


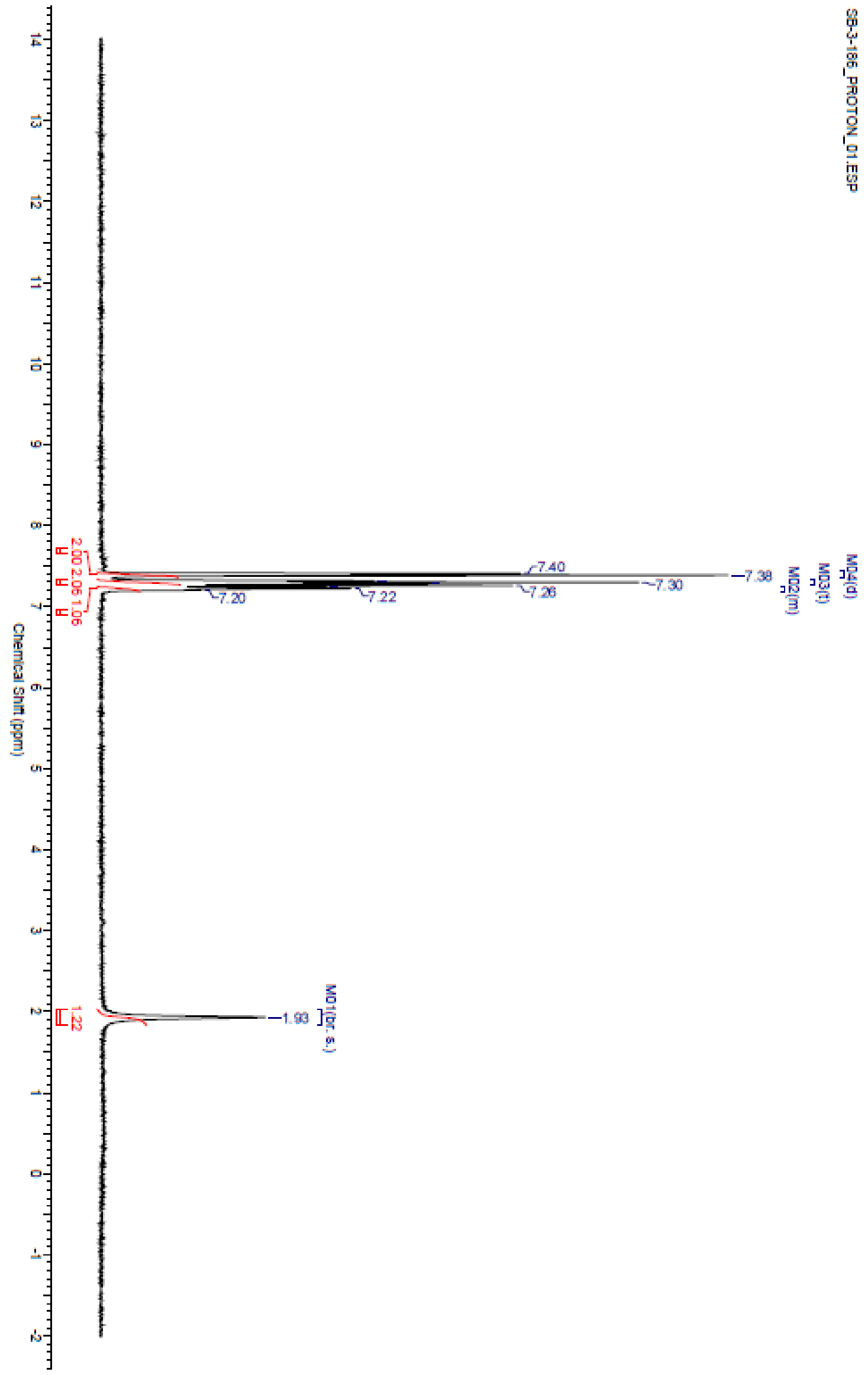

Figure S1: ${ }^{1} \mathrm{H}-\mathrm{NMR}$ of 2-Phenylthio ethanol-1,1,2,2- $\mathrm{D}_{4} \mathbf{1 a}$ 


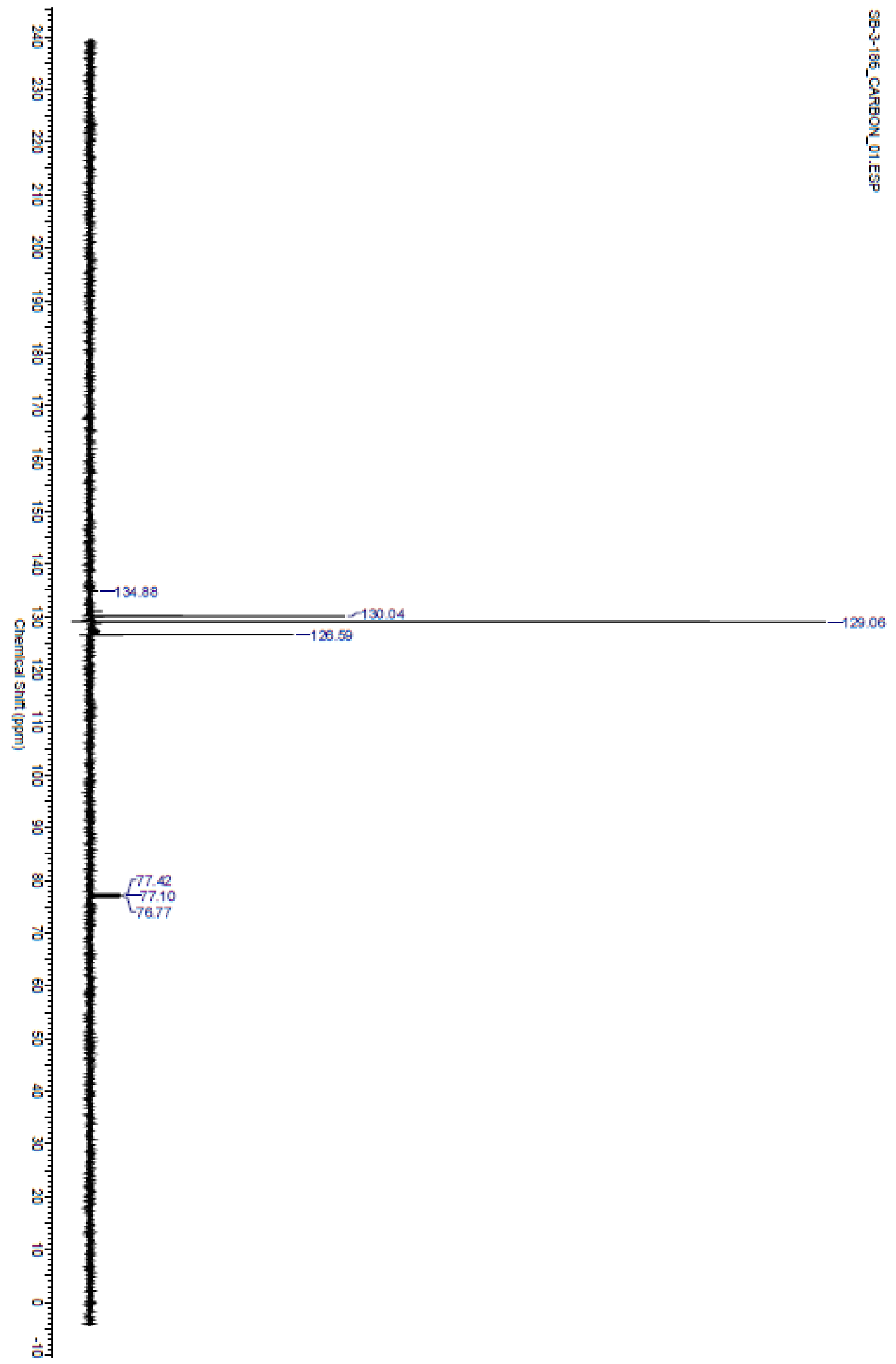

Figure S2: ${ }^{13} \mathrm{C}-\mathrm{NMR}$ of 2-Phenylthio ethanol-1,1,2,2-D 4 1a 


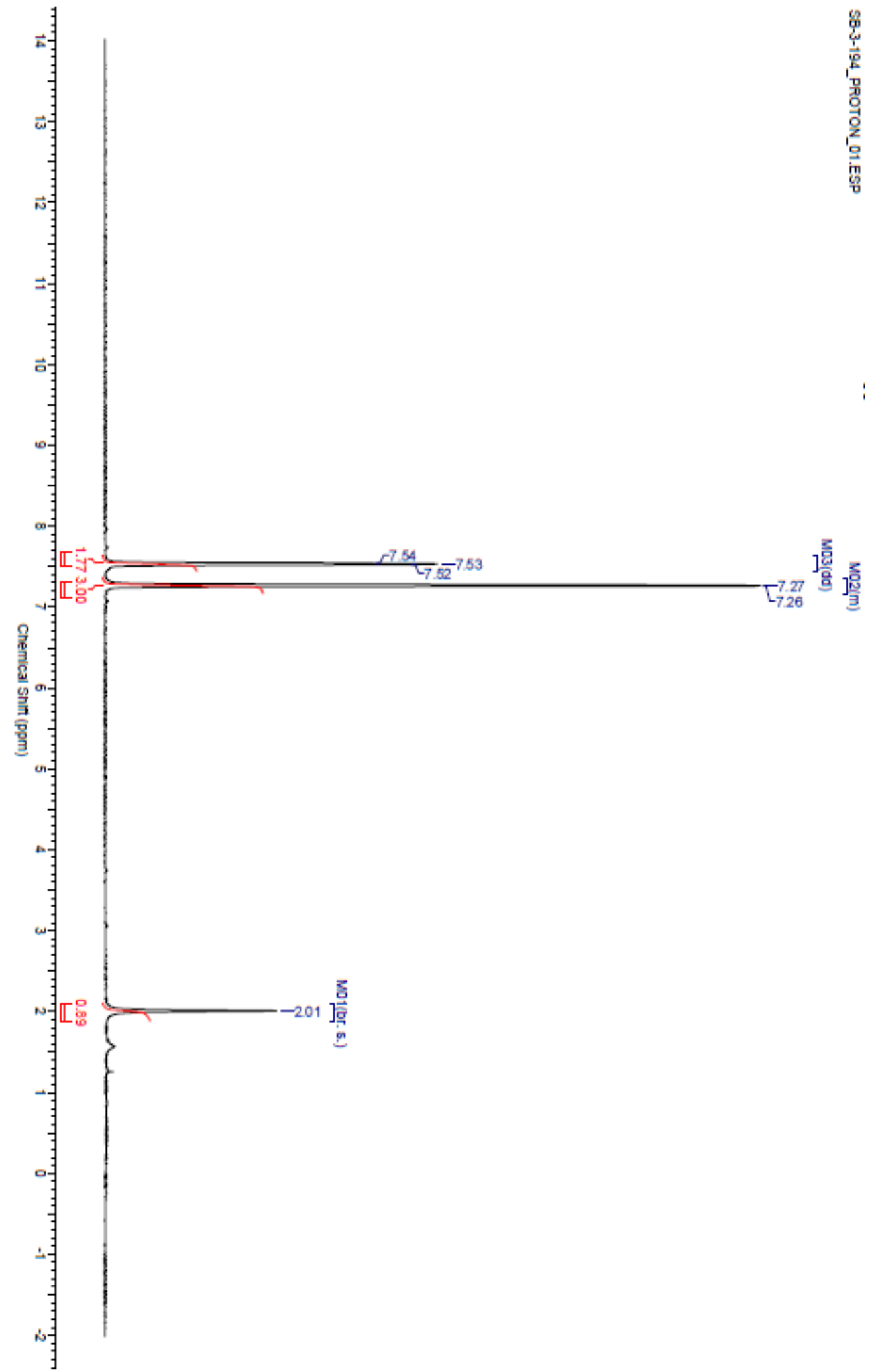

Figure S3: ${ }^{1} \mathrm{H}-\mathrm{NMR}$ of 2-Phenylseleno ethanol-1,1,2,2- $\mathrm{D}_{4}$ 2a 


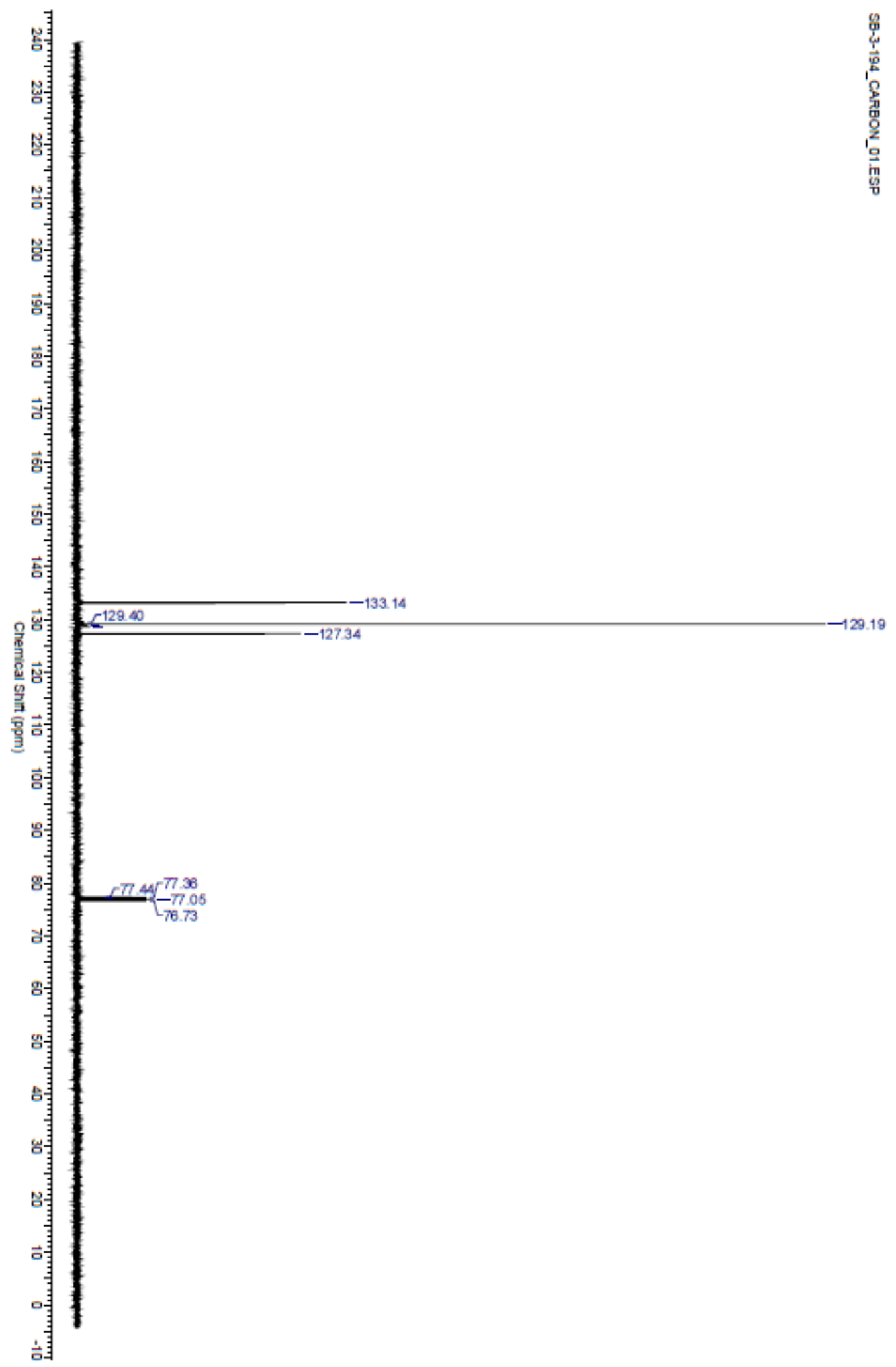

Figure S4: ${ }^{13} \mathrm{C}-\mathrm{NMR}$ of 2-Phenylseleno ethanol-1,1,2,2- $\mathrm{D}_{4} \mathbf{2 a}$

S8 


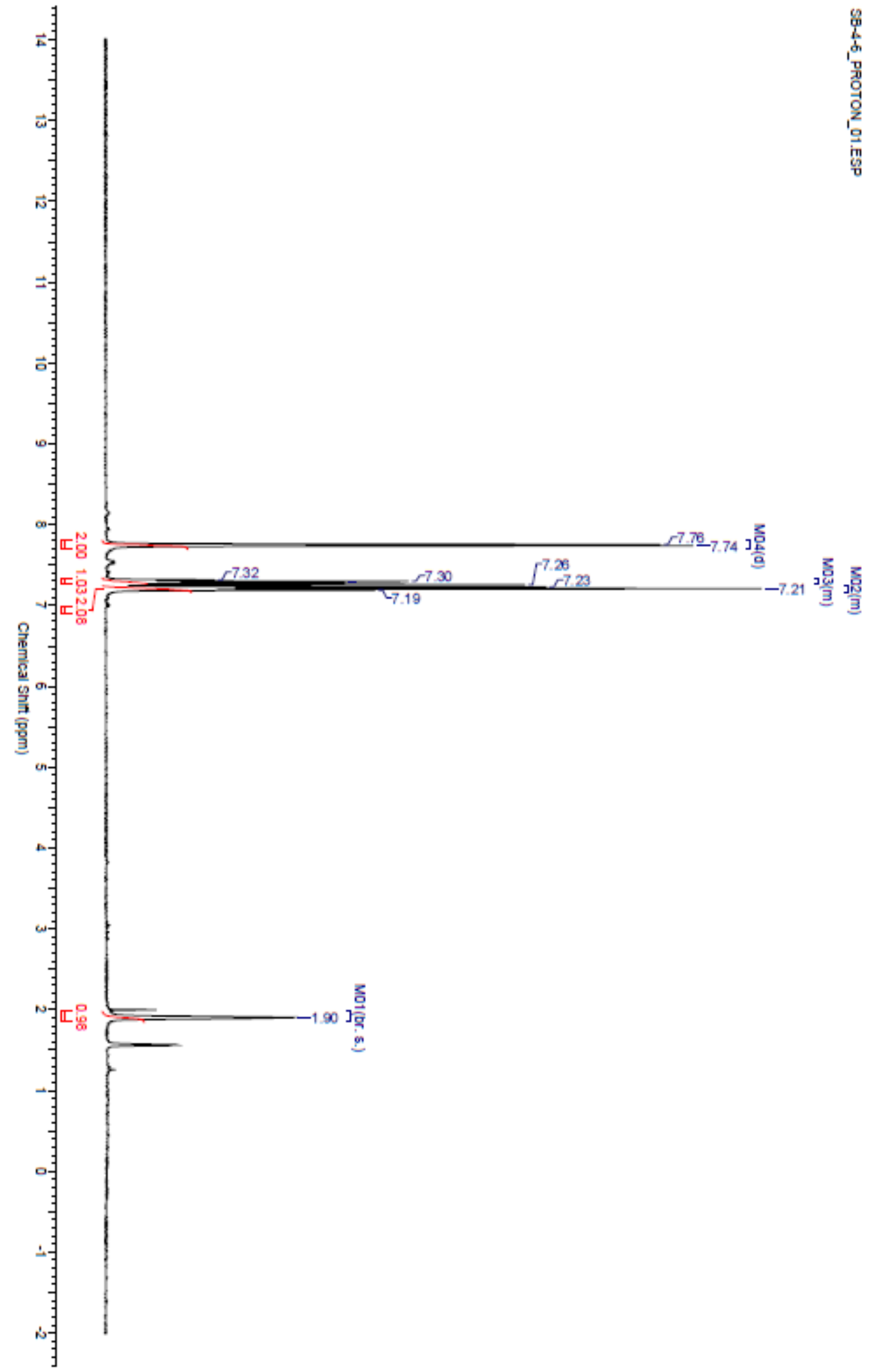

Figure S5: ${ }^{1} \mathrm{H}-\mathrm{NMR}$ 2-Phenyltelluro ethanol-1,1,2,2-D 43 


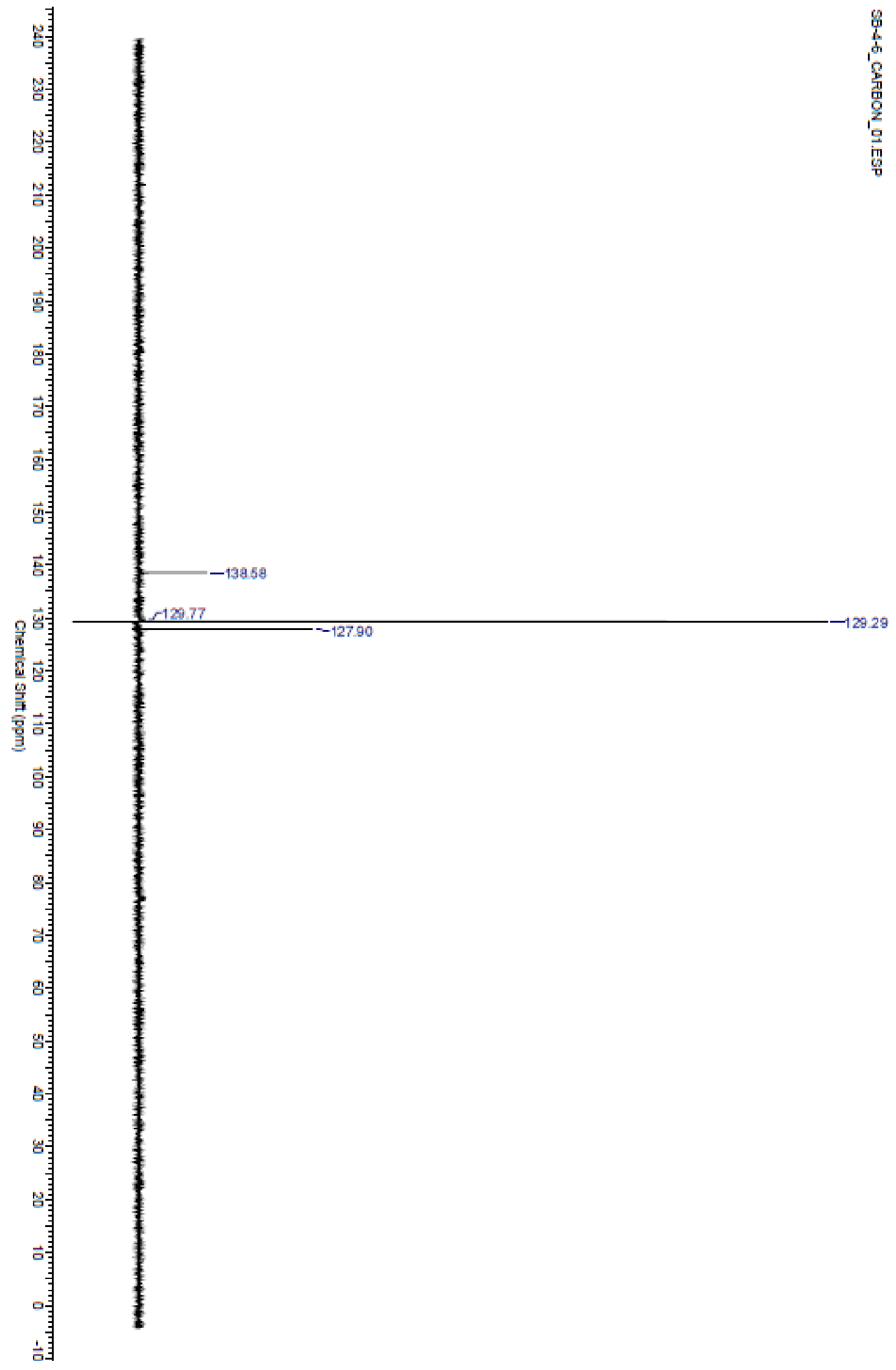

Figure S6: ${ }^{13} \mathrm{C}-\mathrm{NMR}$ of 2-Phenyltelluro ethanol-1,1,2,2-- $\mathrm{D}_{4} 3$ 


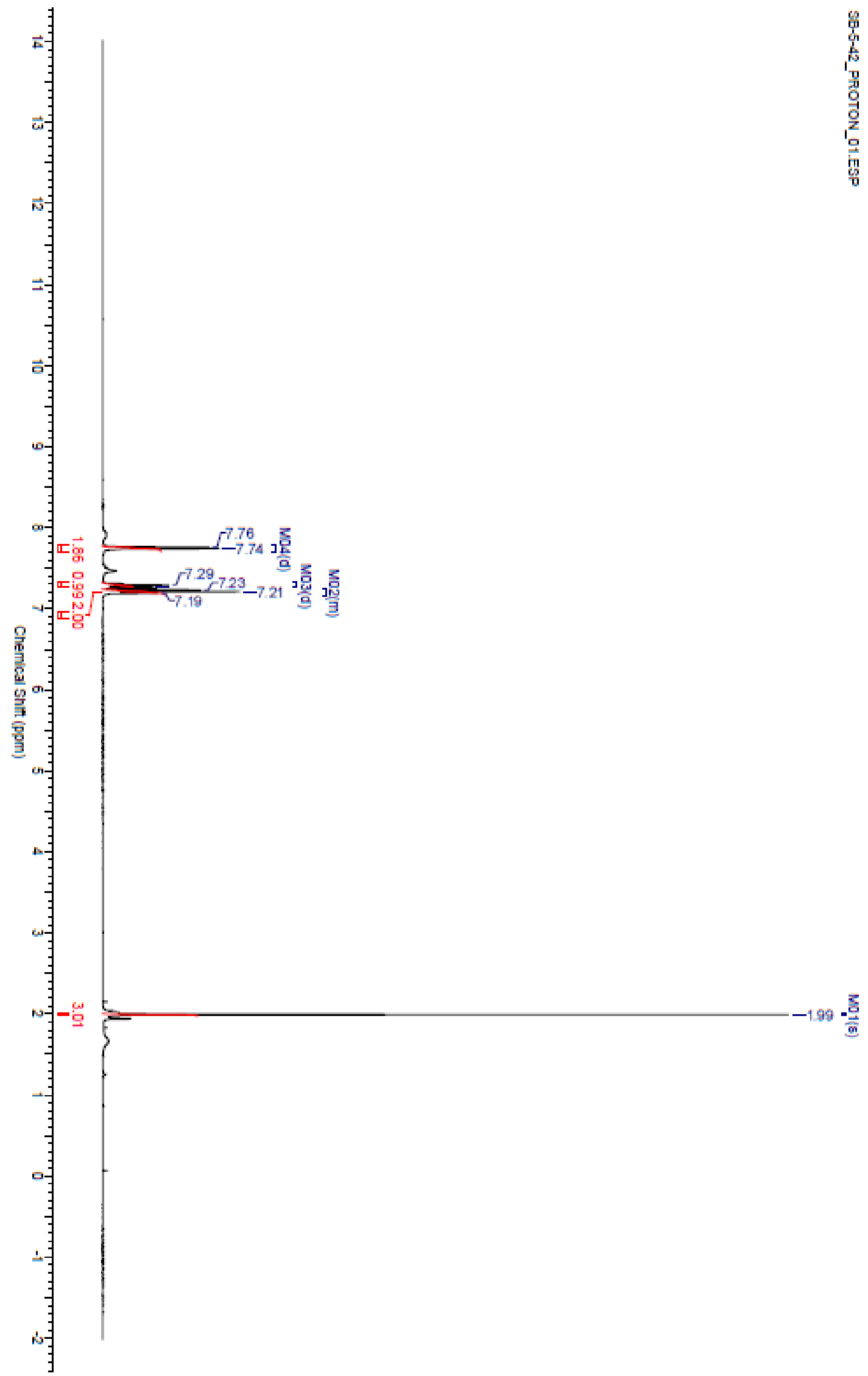

Figure S7: ${ }^{1} \mathrm{H}-\mathrm{NMR}$ 2-(Phenyltelluro)ethyl acetate-1,2,2,2- $\mathrm{D}_{4}$ 3a 


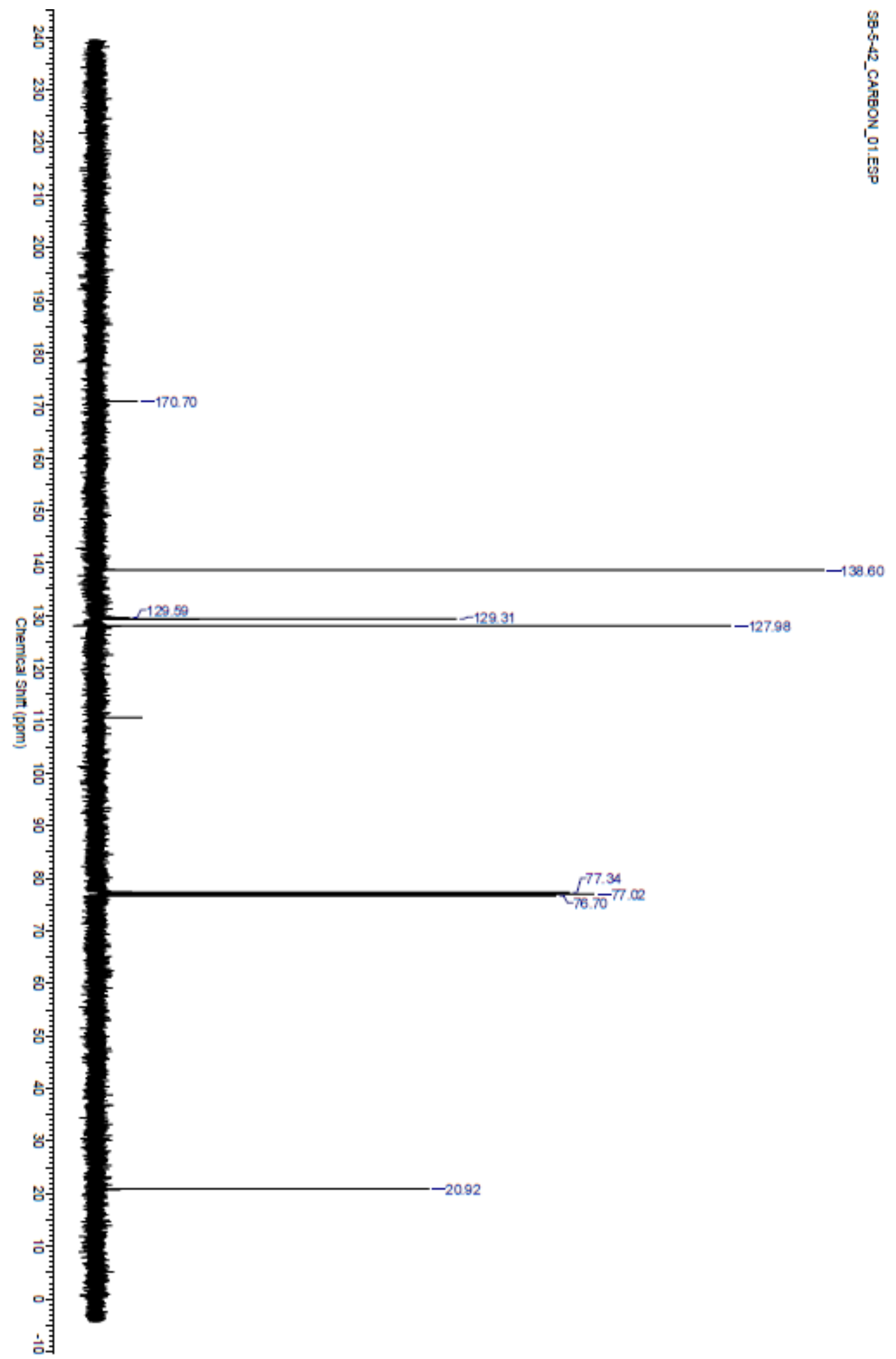

Figure S8: ${ }^{13} \mathrm{C}-\mathrm{NMR}$ of 2-(Phenyltelluro)ethyl acetate-1,2,2,2- $\mathrm{D}_{4} \mathbf{3 a}$ 


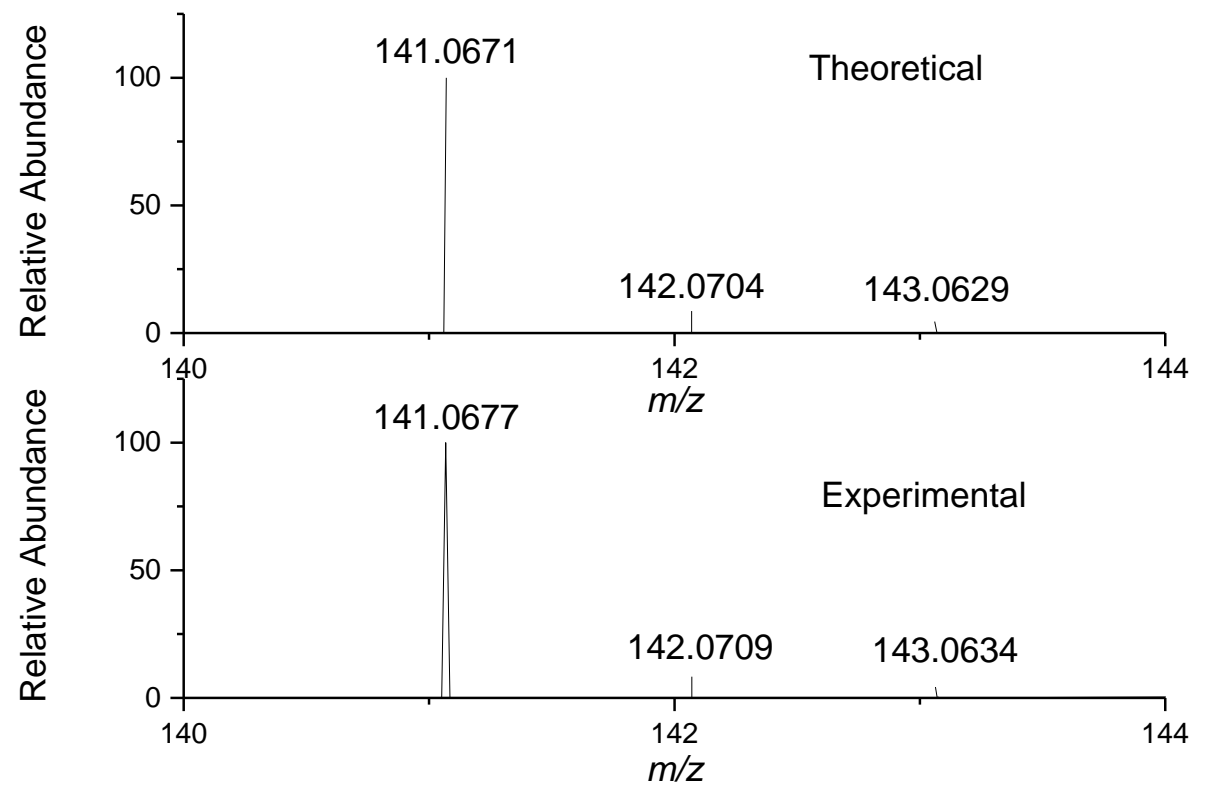

Figure S9. Experimental and theoretical high-resolution accurate mass measurements for the ions corresponding to thiiranium ion $\mathbf{1 b}\left[\mathrm{C}_{8} \mathrm{H}_{5} \mathrm{D}_{4} \mathrm{~S}\right]^{+}(\mathrm{m} / z$ 141). The $m / z$ used is represented on the most intense sulphur isotope $\left({ }^{32} \mathrm{~S}\right)$. The full isotopic envelope is shown.

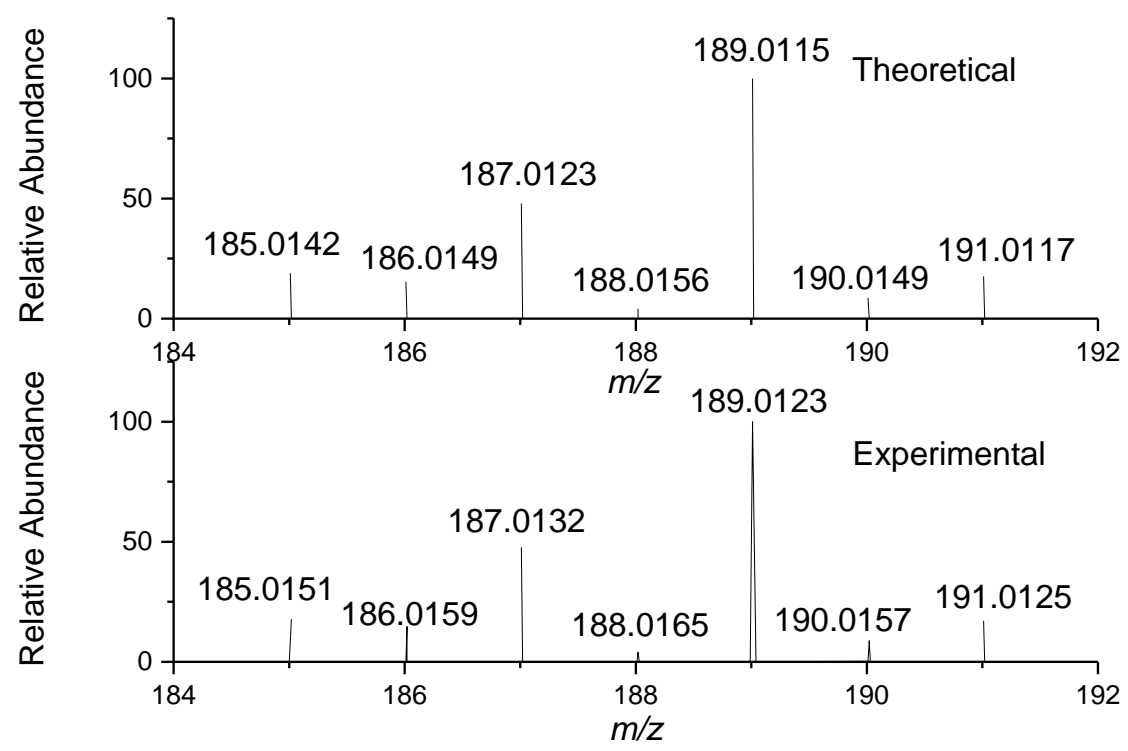

Figure S10. Experimental and theoretical high-resolution accurate mass measurements for the ions corresponding to seleniranium ion $\mathbf{2 b}\left[\mathrm{C}_{8} \mathrm{H}_{5} \mathrm{D}_{4} \mathrm{Se}^{+}(\mathrm{m} / z\right.$ 189). The $m / z$ used is represented on the most intense selenium isotope $\left({ }^{80} \mathrm{Se}\right)$. The full isotopic envelope is shown. 


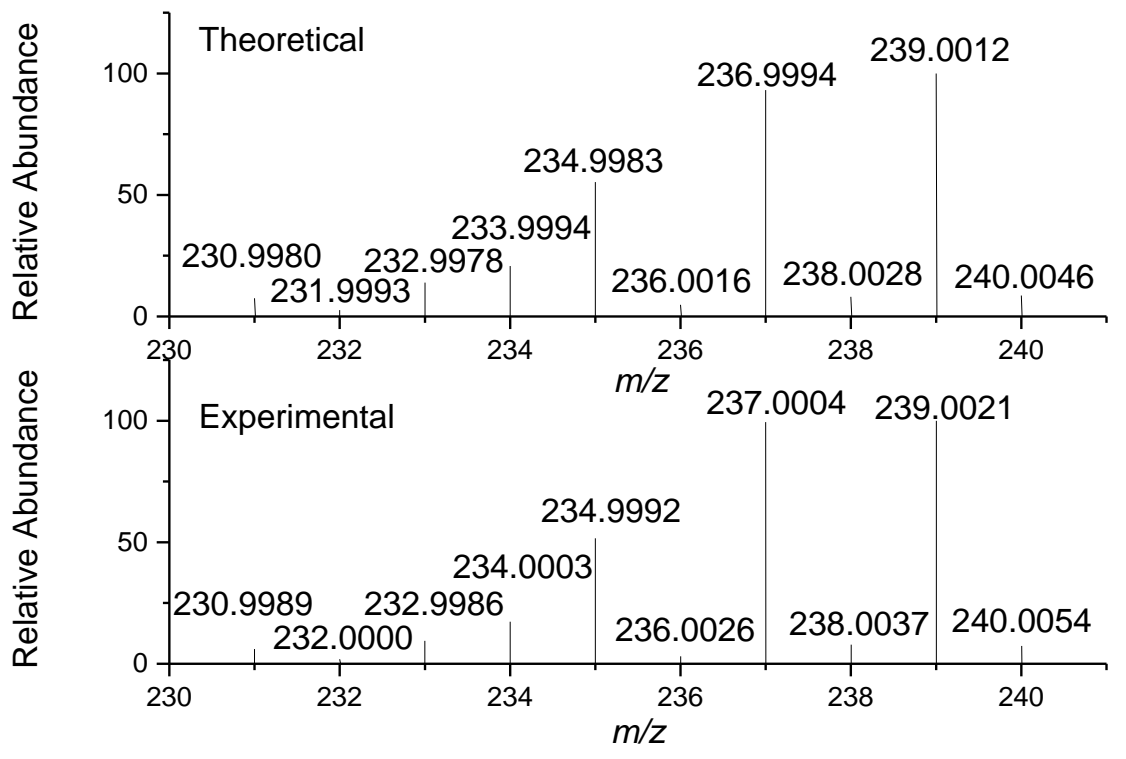

Figure S11. Experimental and theoretical high-resolution accurate mass measurements for the ions corresponding to telluriranium ion $\mathbf{3 b}\left[\mathrm{C}_{8} \mathrm{H}_{5} \mathrm{D}_{4} \mathrm{Te}\right]^{+}(\mathrm{m} / z$ 239). The $\mathrm{m} / z$ used is represented on the most intense tellurium isotope $\left({ }^{130} \mathrm{Te}\right)$. The full isotopic envelope is shown.

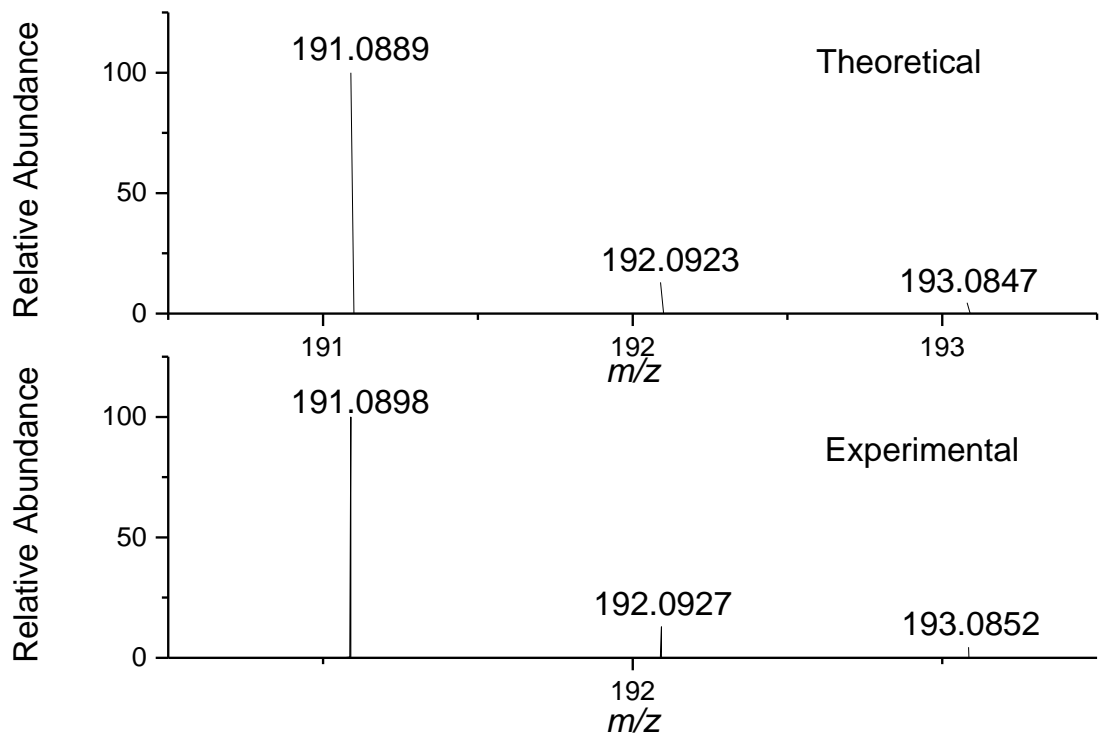

Figure S12. Experimental and theoretical high-resolution accurate mass measurements for the ions corresponding to thiiranium ion $\mathbf{4} \mathbf{b}\left[\mathrm{C}_{12} \mathrm{H}_{15} \mathrm{~S}\right]^{+}(\mathrm{m} / z, 191)$. The $\mathrm{m} / z$ used is represented on the most intense sulphur isotope $\left({ }^{32} \mathrm{~S}\right)$. The full isotopic envelope is shown. 


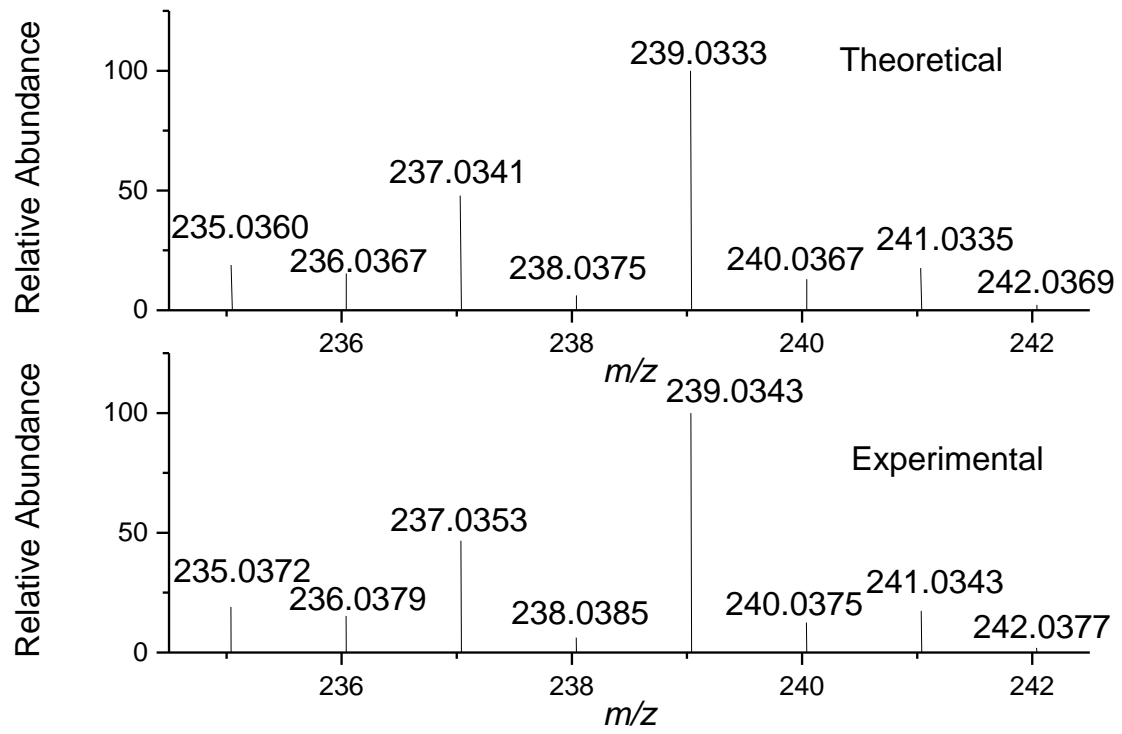

Figure S13. Experimental and theoretical high-resolution accurate mass measurements for the ions corresponding to seleniranium ion $\mathbf{5 b}\left[\mathrm{C}_{12} \mathrm{H}_{15} \mathrm{Se}\right]^{+}(\mathrm{m} / \mathrm{z} 239)$. The $\mathrm{m} / z$ used is represented on the most intense selenium isotope $\left({ }^{80} \mathrm{Se}\right)$. The full isotopic envelope is shown.

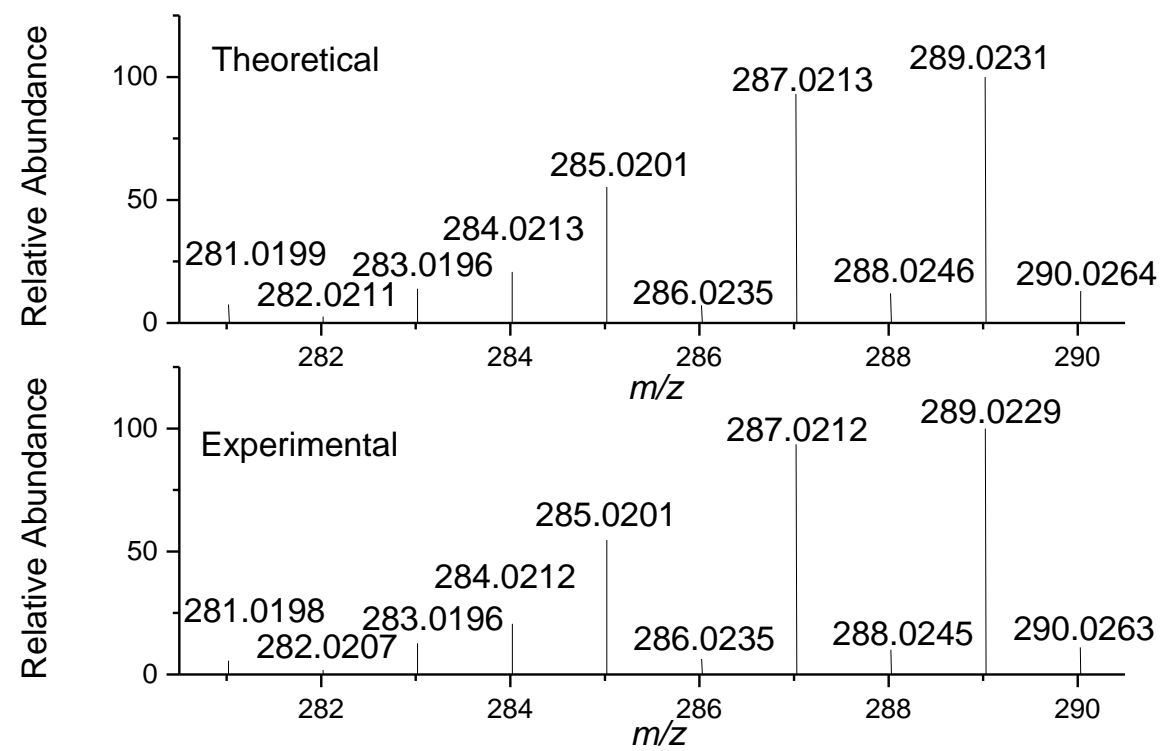

Figure S14. Experimental and theoretical high-resolution accurate mass measurements for the ions corresponding to telluriranium ion $6 \mathbf{b}\left[\mathrm{C}_{12} \mathrm{H}_{15} \mathrm{Te}\right]^{+}(\mathrm{m} / z, 289)$. The $\mathrm{m} / z$ used is represented on the most intense tellurium isotope $\left({ }^{130} \mathrm{Te}\right)$. The full isotopic envelope is shown. 
Time (s)

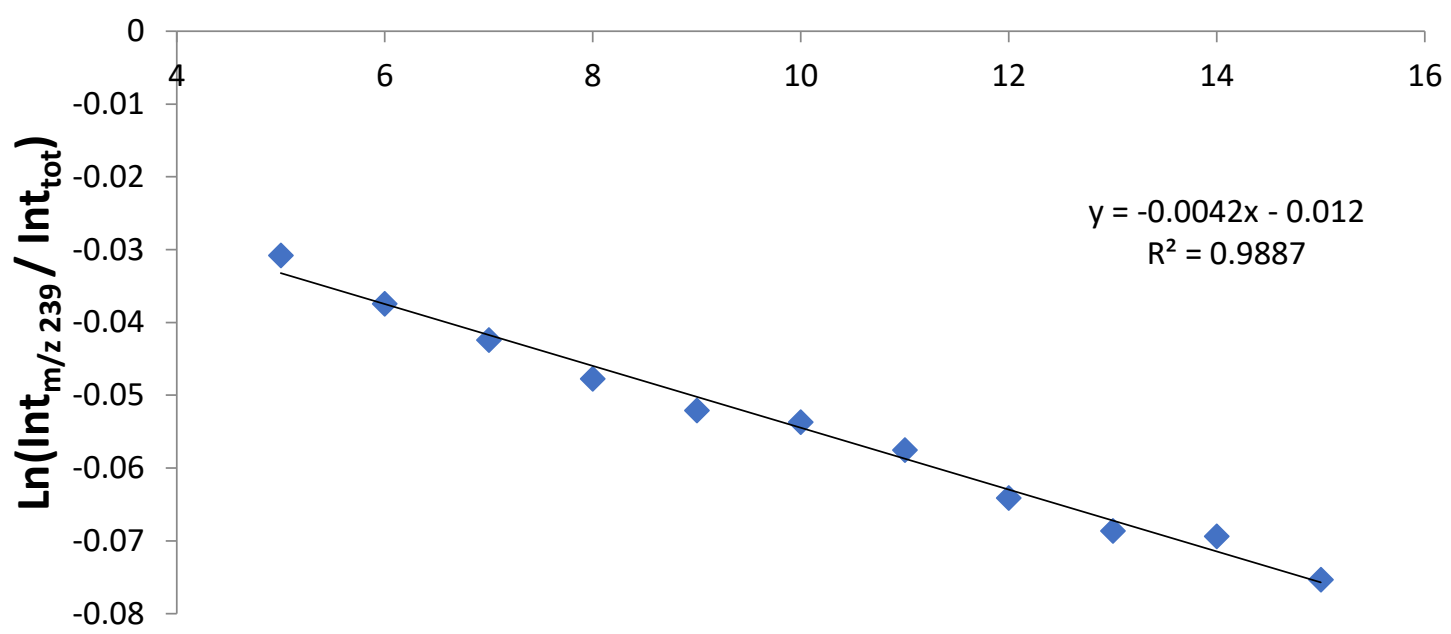

Figure S15: Early onset of the reaction of telluriranium ion $\mathbf{3 b}(\mathrm{m} / z 239)$ with $\mathrm{C}_{2} \mathrm{H}_{4}$ (conc. $c a 3.3 \mathrm{x}$ $10^{10}$ molecule $\mathrm{cm}^{-3}$ ) in the ion trap mass spectrometer in the formation of exchange product $\mathrm{m} / \mathrm{z} 235$.

Time (s)

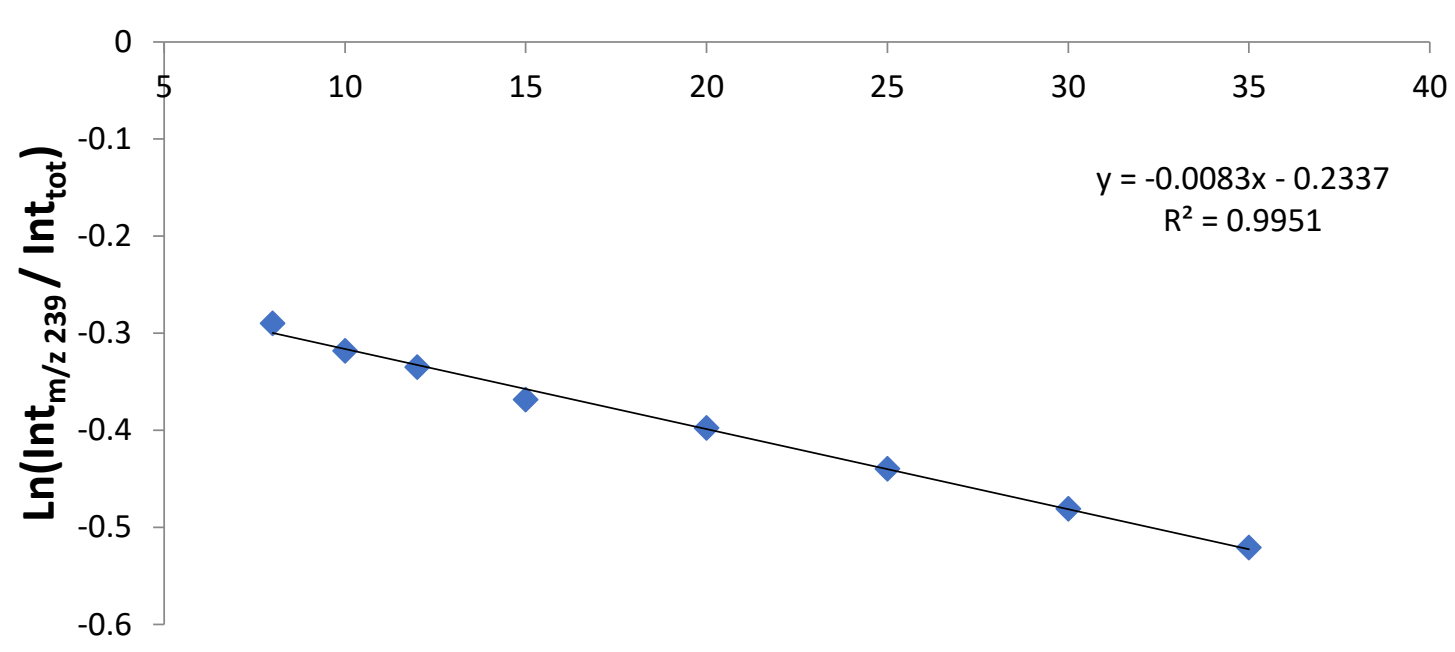

Figure S16: Early onset of the reaction of seleniranium ion $\mathbf{5 b}\left(\mathrm{m} / z\right.$ 239) with $c-\mathrm{C}_{6} \mathrm{D}_{10}$ (conc. $c a 7.2$ $\mathrm{x} 10^{10}$ molecule $\mathrm{cm}^{-3}$ ) in the ion trap mass spectrometer in the formation of exchange product $\mathrm{m} / \mathrm{z} 249$. 


\section{Time (s)}

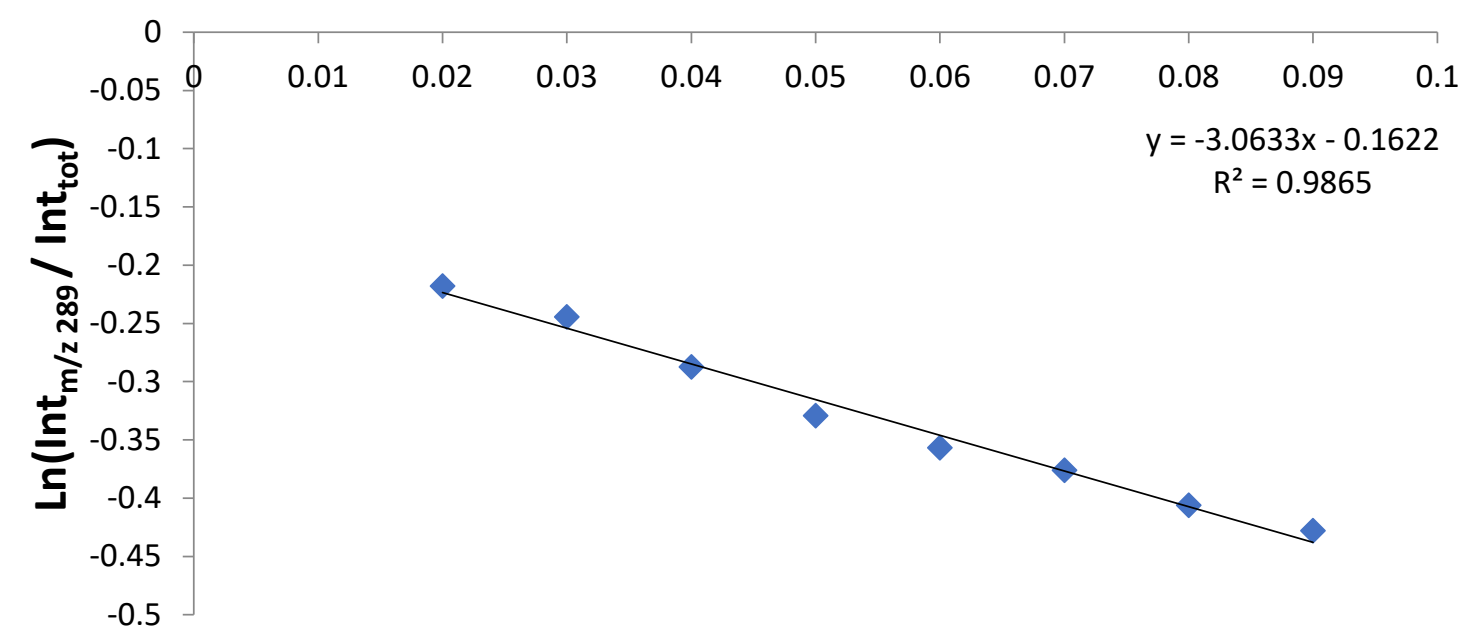

Figure S17: Early onset of the reaction of telluriranium ion $\mathbf{6 b}\left(\mathrm{m} / z\right.$ 289) with $c-\mathrm{C}_{6} \mathrm{D}_{10}$ (conc. $c a 7.2$ $\mathrm{x} 10^{10}$ molecule $\mathrm{cm}^{-3}$ ) in the ion trap mass spectrometer in the formation of exchange product $\mathrm{m} / \mathrm{z} 299$.

Table S1. Sensitivity test of the exchange rate constants predicted by RRKM theory towards variation of the product and barrier energies by $\pm 5 \mathrm{~kJ} \mathrm{~mol}^{-1}$

\begin{tabular}{ccccc}
\multicolumn{5}{c}{$\boldsymbol{k}_{\mathbf{R R K M}}$} \\
\hline Unchanged & Product $+5^{(\mathrm{a})}$ & Product $-5^{(\mathrm{a})}$ & Transition State $+5^{(\mathrm{a})}$ & Transition State $-5^{(\mathrm{a})}$ \\
$\mathbf{2 . 5 E}-12$ & $2.1 \mathrm{E}-12$ & $2.6 \mathrm{E}-12$ & $3.8 \mathrm{E}-13$ & $1.4 \mathrm{E}-11$ \\
$\mathbf{1 . 4 E}-14$ & $1.4 \mathrm{E}-14$ & $1.4 \mathrm{E}-14$ & $1.8 \mathrm{E}-15$ & $1.0 \mathrm{E}-13$ \\
$\mathbf{5 . 3 E}-10$ & $1.6 \mathrm{E}-10$ & $7.6 \mathrm{E}-10$ & $4.4 \mathrm{E}-10$ & $5.8 \mathrm{E}-10$ \\
\hline
\end{tabular}

(a) Variation in energies by $\pm 5 \mathrm{~kJ} \mathrm{~mol}^{-1}$ 


\section{Gaussian Cartesian Coordinates and Energies Calculated at M06-2X/def2-TZVP:}

Energies given as sum of electronic and zero-point vibrational energies (E), sum of electronic and thermal enthalpies $(\mathrm{H})$, sum of electronic and thermal Free energies $(\mathrm{G})$, and zero-point vibrational energy (ZPVE) in Hartrees

\section{Ethene}

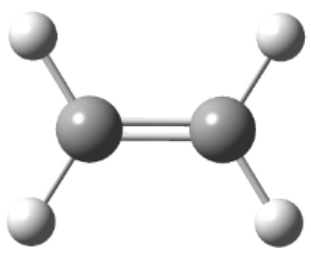

$E=-78.520565$

$\mathrm{H}=-78.516586$

$\mathrm{G}=-78.542078$

$\mathrm{ZPVE}=0.051412$

Symbol

$\mathrm{X}$

Y

Z

$\begin{array}{lrrr}\mathrm{C} & 0.00000000 & -0.66100900 & 0.00000000 \\ \mathrm{H} & -0.92229300 & -1.22855200 & 0.00000000 \\ \mathrm{H} & 0.92225200 & -1.22859300 & 0.00000000 \\ \mathrm{C} & 0.00000000 & 0.66100900 & 0.00000000 \\ \mathrm{H} & 0.92229300 & 1.22855200 & 0.00000000 \\ \mathrm{H} & -0.92225200 & 1.22859300 & 0.00000000\end{array}$

Thiiranium Reactant Ion 1b

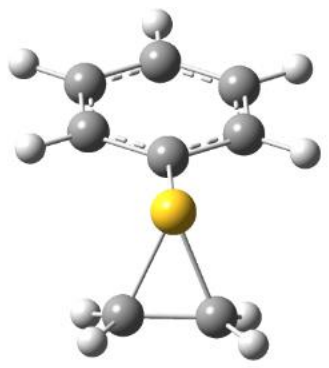

$E=-708.007163$

$H=-707.997675$

$\mathrm{G}=-708.041201$

$\mathrm{ZPVE}=0.135137$

Symbol

X

Y

Z

C

$-2.39654700$

0.73315200

0.71080700 


$\begin{array}{lrrr}\text { H(ISO=2) } & -1.71044500 & 1.25765500 & 1.36231800 \\ \text { H(ISO=2) } & -3.29637800 & 1.26141900 & 0.42366400 \\ \mathrm{C} & -2.39644400 & -0.73208100 & 0.71192000 \\ \mathrm{H}(\text { ISO=2) } & -1.71025000 & -1.25549400 & 1.36421000 \\ \mathrm{H}(\text { ISO=2) } & -3.29620400 & -1.26090600 & 0.42558400 \\ \mathrm{C} & 0.15865900 & -0.00001600 & -0.27594300 \\ \mathrm{C} & 0.81682200 & -1.21762500 & -0.15410800 \\ \mathrm{C} & 0.81659700 & 1.21784500 & -0.15546800 \\ \mathrm{C} & 2.17419500 & -1.20662100 & 0.12699200 \\ \mathrm{H} & 0.28761200 & -2.15446600 & -0.27700000 \\ \mathrm{C} & 2.17397400 & 1.20740100 & 0.12564700 \\ \mathrm{H} & 0.28722500 & 2.15445400 & -0.27941900 \\ \mathrm{C} & 2.84719100 & 0.00053200 & 0.26737300 \\ \mathrm{H} & 2.70508700 & -2.14287000 & 0.23198400 \\ \mathrm{H} & 2.70469400 & 2.14386400 & 0.22959700 \\ \mathrm{H} & 3.90686100 & 0.00075200 & 0.48477800 \\ \mathrm{~S} & -1.55380300 & -0.00049500 & -0.74239200\end{array}$

Association Complex 1c

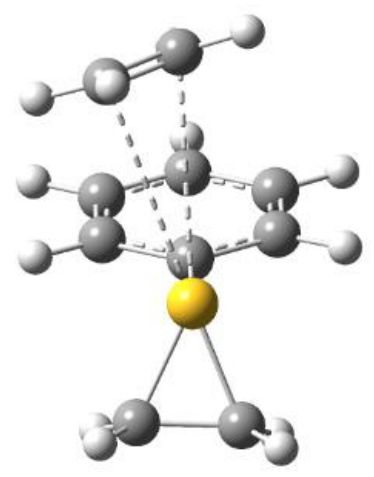

$E=-786.534297$

$H=-786.519902$

$G=-786.577525$

$\mathrm{ZPVE}=0.187573$

Symbol

X

$-1.75491900$

$-1.76108100$

$-0.93760900$

$-0.92717900$

$-2.69503900$

$-2.70631800$

0.05502800

$-0.26489900$

1.09038000

$-0.76964400$

$-0.43735700$

$-1.79510000$

0.47101900
Y

$-1.80963900$

$-2.01947000$

$-2.55167800$

$-2.19231400$

$-1.68005300$

$-2.04048000$

2.90953900

3. 23124700

2.60352400

2.92442100

2.64062100

3.26063200

$-0.35989600$
$-0.84771700$

0.60045900

1.05694800

$-1.42942900$

$-1.36761300$

1.12669300

0.30095900

1. 28487400

0.19922300

$-0.73570600$

$-1.72822600$

$-0.64218100$

0.11735600 


$\begin{array}{lrrr}\text { C } & 1.15498700 & -0.21621900 & -1.08216800 \\ \text { C } & 1.12050000 & -0.40274500 & 1.34323000 \\ \text { C } & 2.53870700 & -0.14094400 & -1.04723500 \\ \text { H } & 0.62362200 & -0.15288700 & -2.02375700 \\ \text { C } & 2.50528400 & -0.32466800 & 1.35906200 \\ \text { H } & 0.56315900 & -0.48830500 & 2.26788700 \\ \text { C } & 3.20925900 & -0.19425800 & 0.16891400 \\ \text { H } & 3.09168600 & -0.03482300 & -1.97058400 \\ \text { H } & 3.03150600 & -0.36224000 & 2.30309000 \\ \text { H } & 4.28894500 & -0.13068500 & 0.18874000 \\ \text { S } & -1.30378700 & -0.30898200 & 0.10763700\end{array}$

Transition State 1d

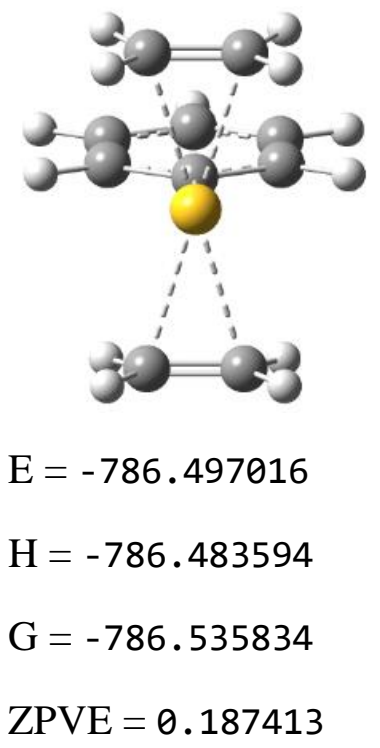

Imaginary Frequency $=-333.9297 \mathrm{~cm}^{-1}$

Symbol

X

Y

$\mathrm{Z}$

$\begin{array}{lrrr}\text { C } & 1.25627900 & -2.28137800 & 0.67843600 \\ \mathrm{C} & 1.25641400 & -2.28138900 & -0.67815900 \\ \mathrm{H}(\text { Iso=2) } & 0.33555100 & -2.37729400 & -1.23870200 \\ \mathrm{H}(\text { Iso=2) } & 0.33530500 & -2.37727300 & 1.23879800 \\ \mathrm{H}(\text { Iso=2) } & 2.18270200 & -2.28958600 & 1.23839400 \\ \mathrm{H}(\text { Iso=2) } & 2.18294700 & -2.28960700 & -1.23793300 \\ \mathrm{C} & 1.25641400 & 2.28137600 & -0.67820200 \\ \mathrm{H} & 2.18294700 & 2.28958400 & -1.23797600 \\ \mathrm{H} & 0.33555100 & 2.37727000 & -1.23874700 \\ \mathrm{C} & 1.25627900 & 2.28139100 & 0.67839200 \\ \mathrm{H} & 0.33530500 & 2.37729600 & 1.23875300 \\ \mathrm{H} & 2.18270200 & 2.28961000 & 1.23835000 \\ \mathrm{C} & -0.59987900 & 0.00000000 & -0.00005700 \\ \mathrm{C} & -1.28679400 & 0.00001200 & 1.21101600 \\ \mathrm{C} & -1.28657200 & -0.00001200 & -1.21125600 \\ \mathrm{C} & -2.67299900 & 0.00001200 & 1.20561600 \\ \mathrm{H} & -0.74040400 & 0.00002100 & 2.14642600 \\ \mathrm{C} & -2.67277700 & -0.00001200 & -1.20611100\end{array}$




$\begin{array}{rrrr}H & -0.74000800 & -0.00002100 & -2.14656600 \\ \mathrm{C} & -3.36323200 & 0.00000000 & -0.00031100 \\ \mathrm{H} & -3.21335700 & 0.00002100 & 2.14256000 \\ \mathrm{H} & -3.21296300 & -0.00002100 & -2.14315500 \\ \mathrm{H} & -4.44509500 & 0.00000000 & -0.00041000 \\ \mathrm{~S} & 1.17804100 & 0.00000000 & 0.00010900\end{array}$

Dissociation Complex 1e

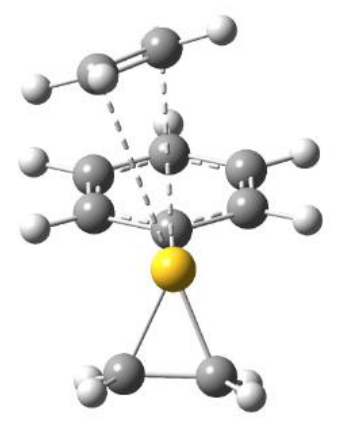

$E=-786.533543$

$H=-786.519213$

$G=-786.577151$

$\mathrm{ZPVE}=0.188327$

Symbol

X

Y

Z

$\mathrm{C}$
$\mathrm{C}$
$\mathrm{H}$
$\mathrm{H}$
$\mathrm{H}$
$\mathrm{H}$
$\mathrm{C}$
$\mathrm{H}(\mathrm{IsO}=2)$
$\mathrm{H}(\mathrm{IsO}=2)$
$\mathrm{C}$
$\mathrm{H}(\mathrm{IsO}=2)$
$\mathrm{H}(\mathrm{IsO}=2)$
$\mathrm{C}$
$\mathrm{C}$
$\mathrm{C}$
$\mathrm{C}$
$\mathrm{H}$
$\mathrm{C}$
$\mathrm{H}$
$\mathrm{C}$
$\mathrm{H}$
$\mathrm{H}$
$\mathrm{H}$
$\mathrm{S}$

$-1.75491900$

$-1.80963900$

$-0.84771700$

$-1.76108100$

$-2.01947000$

0.60045900

$-0.93760900$

$-2.55167800$

1.05694800

$-0.92717900$

$-2.19231400$

$-1.42942900$

$-2.69503900$

$-1.68005300$

$-1.36761300$

$-2.70631800$

$-2.04048000$

1.12669300

0.05502800

2.90953900

0.30095900

$-0.26489900$

3.23124700

1.28487400

1.09038000

2.60352400

0.19922300

$-0.76964400$

2.92442100

$-0.73570600$

$-0.43735700$

2. 64062100

$-1.72822600$

$-1.79510000$

3. 26063200

$-0.64218100$

0.47101900

$-0.35989600$

0.11735600

1.15498700

$-0.21621900$

$-1.08216800$

1.12050000

$-0.40274500$

1.34323000

2.53870700

$-0.14094400$

$-1.04723500$

0.62362200

$-0.15288700$

$-2.02375700$

2.50528400

$-0.32466800$

1. 35906200

0.56315900

$-0.48830500$

2. 26788700

3.20925900

$-0.19425800$

0.16891400

3.09168600

$-0.03482300$

$-1.97058400$

3.03150600

$-0.36224000$

2. 30309000

4.28894500

$-0.13068500$

0.18874000

$-1.30378700$

$-0.30898200$

0.10763700 


\section{Thiiranium Product Ion 1f}

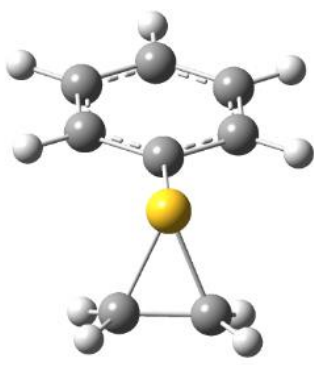

$E=-707.993884$

$H=-707.984905$

$\mathrm{G}=-708.027462$

$\mathrm{ZPVE}=0.148416$

Symbol

X

Y

Z

C

$\mathrm{H}$

$-2.39654700$

0.73315200

0.71080700

$-1.71044500$

1.25765500

1.36231800

$-3.29637800$

1.26141900

0.42366400

$-2.39644400$

$-0.73208100$

0.71192000

$-1.71025000$

$-1.25549400$

1.36421000

$-3.29620400$

$-1.26090600$

0.42558400

0.15865900

$-0.00001600$

$-0.27594300$

0.81682200

$-1.21762500$

$-0.15410800$

0.81659700

1.21784500

$-0.15546800$

2.17419500

$-1.20662100$

0.12699200

0.28761200

$-2.15446600$

$-0.27700000$

2.17397400

1. 20740100

0.12564700

0.28722500

2.15445400

$-0.27941900$

2.84719100

0.00053200

0.26737300

2.70508700

$-2.14287000$

0.23198400

2.70469400

2.14386400

3.90686100

0.00075200

0.22959700

$-1.55380300$

$-0.00049500$

0.48477800

$-0.74239200$

Ethene-d4

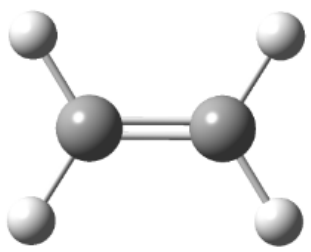

$E=-78.532937$

$\mathrm{H}=-78.528642$

$G=-78.555398$ 
$\mathrm{ZPVE}=0.039040$

Symbol

$\mathrm{X}$

Y

Z

$$
\begin{aligned}
& \mathrm{C} \\
& \mathrm{H}(\text { IsO }=2) \\
& \mathrm{H}(\text { IsO }=2) \\
& \mathrm{C} \\
& \mathrm{H}(\text { IsO }=2) \\
& \mathrm{H}(\text { IsO }=2)
\end{aligned}
$$

0.00000000

$-0.66100900$

0.00000000

$-0.92229300$

$-1.22855200$

0.92225200

$-1.22859300$

0.00000000

0.66100900

0.00000000

0.00000000

0.92229300

1.22855200

0.00000000

$-0.92225200$

1.22859300

0.00000000

0.00000000

Seleniranium Reactant Ion 2b

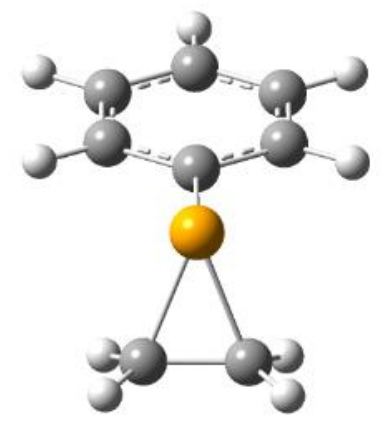

$E=-2711.399023$

$H=-2711.389052$

$\mathrm{G}=-2711.434331$

$\mathrm{ZPVE}=0.133766$

Symbol

X

Y

Z

\section{C}

$\mathrm{H}(\mathrm{IsO}=2)$

C

2.19106700

1.12884000

0.58090400

1.45391200

1.62068100

1.20157100

2.37123400

$-0.30699000$

0.69054300

$\mathrm{H}(\mathrm{IsO}=2)$

1.76579500

$-0.86646800$

1.39126100

$\mathrm{H}(\mathrm{IsO}=2)$

3.00425800

1.73996000

0.21163500

$-0.46698000$

0.00186100

$-0.20036400$

C

$-0.94887900$

$-1.26627900$

0.09824400

$-1.24685100$

1.14390400

$-0.06926600$

$-2.24854500$

$-1.38372600$

0.56810600

$-0.33213800$

$-2.14664100$

$-0.03249500$

$-2.54388200$

1.00564900

0.40223600

$-0.85999600$

2.12125600

$-0.32930500$

$-3.03967000$

$-0.25225400$

0.72020900

$-2.64211300$

$-2.36146100$

0.81055200

$-3.16663800$

1.88249700

0.51605900

$-4.05320100$

$-0.35221000$

1.08490000

3.31640000

$-0.74777000$

0.40169600

1.29870500

0.16828400

$-0.93038300$ 


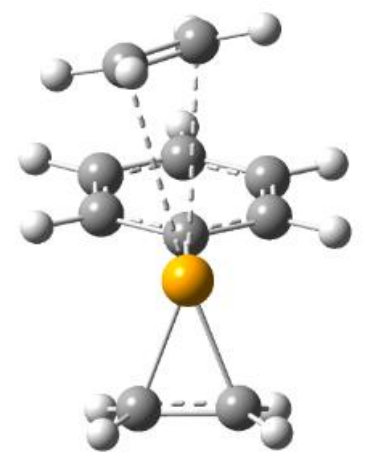

$E=-2789.927580$

$H=-2789.912684$

$G=-2789.971436$

$\mathrm{ZPVE}=0.186138$

Symbol

X Y

$-1.77868200$

$-1.81826900$

$-0.97641500$

$-0.90648800$

$-2.70537900$

$-2.77449700$

$-0.02243900$

$-0.38556300$

1.01936800

$-0.80494600$

$-0.42901300$

$-1.83680100$

0.52104000

1.22634800

1.15461600

2.61268900

0.71225400

2.54205600

0.58615700

3.26593400

3.18069500

3.05469800

4.34720000

$-1.39341300$
$-1.96322900$

$-2.03618600$

$-2.45379900$

$-2.32428600$

$-1.92439800$

$-2.05100700$

2.97553800

3.26049300

2. 68410100

3.01893300

2.77368900

3.34314300

$-0.28872900$

$-0.18104400$

$-0.37026500$

$-0.17835400$

$-0.09401600$

$-0.36656900$

$-0.42719700$

$-0.27065500$

$-0.09994800$

$-0.43375100$

$-0.26448100$

$-0.16828300$

\section{Z}

$-0.81631200$

0.62710000

1.16266500

$-1.34463800$

$-1.37353000$

1.13314200

0.36671300

1.34711500

0.29628400

$-0.70259600$

$-1.68981900$

$-0.63975100$

0.05868500

$-1.13204100$

1.29068500

$-1.08159800$

$-2.08125100$

1. 32249500

2. 21060000

0.14116600

$-1.99870200$

2. 27249800

0.17337700

0.00816800 
Transition State 2d

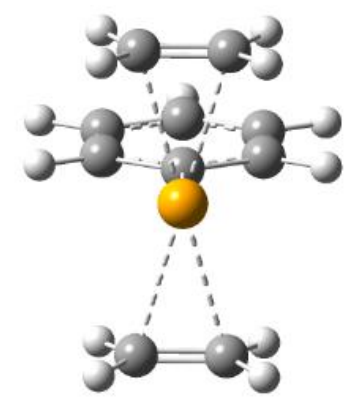

$E=-2789.908173$

$H=-2789.894430$

$\mathrm{G}=-2789.948071$

$\mathrm{ZPVE}=0.186630$

Imaginary Frequency $=-219.2537 \mathrm{~cm}^{-1}$

Symbol

$$
\text { X }
$$

Y

Z

$\begin{array}{lrrr}\text { C } & 1.27356700 & -2.37496800 & 0.67846100 \\ \text { C } & 1.27370100 & -2.37498000 & -0.67817900 \\ \text { H(Iso=2) } & 0.34833200 & -2.42245700 & -1.23824500 \\ \text { H(IsO=2) } & 0.34808700 & -2.42243500 & 1.23834600 \\ \text { H(IsO=2) } & 2.19770000 & -2.43979800 & 1.23899300 \\ \text { H(IsO=2) } & 2.19794500 & -2.43982100 & -1.23852700 \\ \text { C } & 1.27370100 & 2.37496700 & -0.67822400 \\ \text { H } & 2.19794500 & 2.43979700 & -1.23857300 \\ \text { H } & 0.34833200 & 2.42243400 & -1.23829100 \\ \text { C } & 1.27356700 & 2.37498100 & 0.67841600 \\ \text { H } & 0.34808700 & 2.42245800 & 1.23830000 \\ \text { H } & 2.19770000 & 2.43982200 & 1.23894600 \\ \text { C } & -0.62912500 & 0.00000000 & -0.00006000 \\ \text { C } & -1.31201200 & 0.00001200 & 1.21144600 \\ \text { C } & -1.31178900 & -0.00001200 & -1.21169100 \\ \text { C } & -2.69948100 & 0.00001200 & 1.20493100 \\ \text { H } & -0.76940500 & 0.00002100 & 2.14884800 \\ \text { C } & -2.69926000 & -0.00001200 & -1.20543200 \\ \text { H } & -0.76900800 & -0.00002100 & -2.14899200 \\ \text { C } & -3.38979500 & 0.00000000 & -0.00031400 \\ \text { H } & -3.23945700 & 0.00002100 & 2.14220500 \\ \text { H } & -3.23906300 & -0.00002100 & -2.14280500 \\ \text { H } & -4.47161800 & 0.00000000 & -0.00041300 \\ \text { Se } & 1.29370500 & 0.00000000 & 0.00012000\end{array}$




\section{Dissociation Complex 2e}

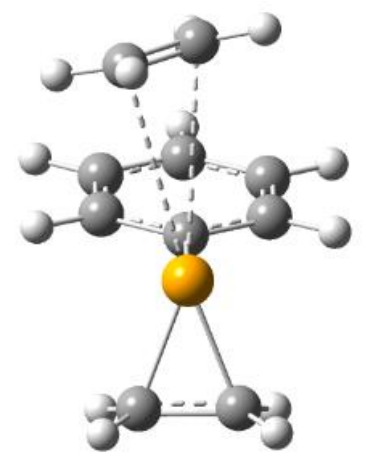

$E=-2789.926863$

$H=-2789.912066$

$G=-2789.971056$

$\mathrm{ZPVE}=0.186855$

Symbol

X

Y

Z

$\mathrm{C}$
$\mathrm{C}$
$\mathrm{H}$
$\mathrm{H}$
$\mathrm{H}$
$\mathrm{H}$
$\mathrm{C}$
$\mathrm{H}(\mathrm{IsO}=2)$
$\mathrm{H}(\mathrm{IsO}=2)$
$\mathrm{C}$
$\mathrm{H}(\mathrm{IsO}=2)$
$\mathrm{H}(\mathrm{IsO}=2)$
$\mathrm{C}$
$\mathrm{C}$
$\mathrm{C}$
$\mathrm{C}$
$\mathrm{H}$
$\mathrm{C}$
$\mathrm{H}$
$\mathrm{C}$
$\mathrm{H}$
$\mathrm{H}$
$\mathrm{H}$
$\mathrm{Se}$

$-1.77868200$

$-1.96322900$

$-0.81631200$

$-1.81826900$

$-2.03618600$

0.62710000

$-0.97641500$

$-2.45379900$

1.16266500

$-0.90648800$

$-2.32428600$

$-1.34463800$

$-2.70537900$

$-1.92439800$

$-1.37353000$

$-2.77449700$

$-2.05100700$

1.13314200

$-0.02243900$

2.97553800

0.36671300

$-0.38556300$

3.26049300

1.34711500

1.01936800

2. 68410100

0.29628400

$-0.80494600$

3.01893300

$-0.70259600$

$-0.42901300$

2.77368900

$-1.68981900$

$-1.83680100$

3.34314300

$-0.63975100$

0.52104000

$-0.28872900$

0.05868500

1.22634800

$-0.18104400$

$-1.13204100$

1.15461600

$-0.37026500$

1.29068500

2.61268900

$-0.17835400$

$-1.08159800$

0.71225400

$-0.09401600$

$-2.08125100$

2.54205600

$-0.36656900$

1. 32249500

0.58615700

$-0.42719700$

2. 21060000

3.26593400

$-0.27065500$

0.14116600

3.18069500

$-0.09994800$

$-1.99870200$

3.05469800

$-0.43375100$

2.27249800

4.34720000

$-0.26448100$

0.17337700

$-1.39341300$

$-0.16828300$

0.00816800 
Seleniranium Product Ion 2f

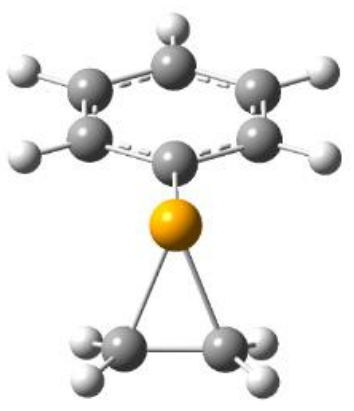

$E=-2711.385787$

$H=-2711.376346$

$\mathrm{G}=-2711.420640$

$\mathrm{ZPVE}=0.147001$

Symbol

X

Y

Z

C

2.19160600

1.12715300

0.58374000

$\mathrm{H}$

1.45477700

1.61705700

1.20633200

C

2.37123200

$-0.30907900$

0.68811900

$\mathrm{H}$

1.76587500

$-0.87079400$

1.38711900

3.00474100

1.73933300

0.21610400

C

$-0.46731800$

0.00392500

$-0.20042000$

$-0.94728700$

$-1.26438900$

0.10038800

C

$-1.24870200$

1.14515700

$-0.07164400$

$-2.24687800$

$-1.38279000$

0.57034400

$-0.32936000$

$-2.14410600$

$-0.02892300$

$-2.54565000$

1.00587200

0.39991700

2.12254300

$-0.33333000$

$-0.86310400$

$-0.25214800$

0.72019900

$-2.63918200$

$-2.36060200$

0.81452900

$-3.16975600$

1.88198000

0.51203700

$-4.05304000$

$-0.35288100$

1.08494600

3.31599500

$-0.74932800$

0.39715600

1.29813100

0.17223200

$-0.93051000$

Telluriranium Reactant Ion 3b

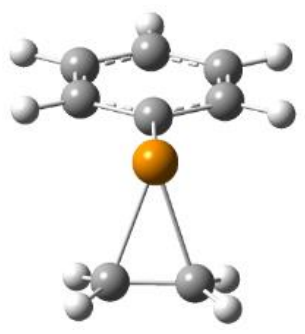

$E=-577.766173$

$H=-577.755782$ 


\begin{tabular}{|c|c|c|c|}
\hline C & 2.30771500 & 1.13623900 & 0.63694800 \\
\hline $\mathrm{H}(\mathrm{ISO}=2)$ & 1.52705500 & 1.62292700 & 1.20753300 \\
\hline C & 2.48535000 & -0.28047200 & 0.74095700 \\
\hline $\mathrm{H}(\mathrm{ISO}=2)$ & 1.83783400 & -0.85675300 & 1.38942200 \\
\hline $\mathrm{H}(\mathrm{IsO}=2)$ & 3.12780400 & 1.75893500 & 0.30299500 \\
\hline C & -0.54342900 & -0.01190400 & -0.27724200 \\
\hline C & -1.02348400 & -1.27556500 & 0.05548800 \\
\hline C & -1.32303000 & 1.12998500 & -0.11491800 \\
\hline C & -2.30303400 & -1.38872600 & 0.57892700 \\
\hline $\mathrm{H}$ & -0.41837100 & -2.16192300 & -0.08925300 \\
\hline C & -2.60032900 & 0.99897500 & 0.4098656 \\
\hline $\mathrm{H}$ & -0.95013300 & 2.10825000 & -0.39174100 \\
\hline C & -3.08605200 & -0.25535500 & 0.75552700 \\
\hline $\mathrm{H}$ & -2.68779100 & -2.36417000 & 0.84424706 \\
\hline $\mathrm{H}$ & -3.21598200 & 1.87814200 & 0.54396806 \\
\hline $\mathrm{H}$ & -4.08436000 & -0.35091500 & 1.16129506 \\
\hline $\mathrm{H}(\mathrm{IsO}=2)$ & 3.43914500 & -0.72406700 & 0.48542200 \\
\hline $\mathrm{Te}$ & 1.36357100 & 0.16553600 & -1.12334000 \\
\hline
\end{tabular}

Association Complex 3c

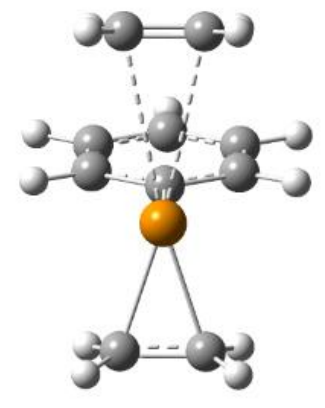

$E=-656.298164$

$H=-656.282997$

$G=-656.342542$

$\mathrm{ZPVE}=0.185299$

Symbol

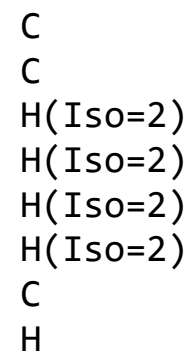

X

$-1.84330300$

$-1.83797300$

$-0.94852400$

$-0.95795100$

$-2.78064100$

$-2.77118700$

$-0.54070800$

$-1.38926500$
$-2.07085000$

$-2.08025900$

$-2.38183800$

$-2.36516500$

$-2.07871600$

$-2.09541400$

3.07510000

3.42476300
Z

$-0.73113800$

0.68140000

1. 21942800

$-1.27983400$

$-1.27233000$

1.22952100

0.66850500

1. 24462800 


$\begin{array}{lrrr}\text { H } & 0.33524800 & 2.75914300 & 1.22401900 \\ \mathrm{C} & -0.55001000 & 3.08432400 & -0.66051800 \\ \mathrm{H} & 0.31809400 & 2.77631400 & -1.23257000 \\ \mathrm{H} & -1.40651000 & 3.44185700 & -1.21980800 \\ \mathrm{C} & 0.60755400 & -0.17303500 & -0.02134800 \\ \mathrm{C} & 1.28343300 & -0.21762400 & -1.23671100 \\ \mathrm{C} & 1.29208800 & -0.23642100 & 1.18833200 \\ \mathrm{C} & 2.66582200 & -0.34139500 & -1.23510300 \\ \mathrm{H} & 0.74760600 & -0.15511900 & -2.17603800 \\ \mathrm{C} & 2.67441200 & -0.36013100 & 1.17495900 \\ \mathrm{H} & 0.76301500 & -0.18831600 & 2.13231400 \\ \mathrm{C} & 3.35729400 & -0.41288700 & -0.03299200 \\ \mathrm{H} & 3.20061500 & -0.38008900 & -2.17462300 \\ \mathrm{H} & 3.21588500 & -0.41338800 & 2.10993600 \\ \mathrm{H} & 4.43493800 & -0.50789900 & -0.03756900 \\ \mathrm{Te} & -1.47878400 & 0.05674300 & -0.01200400\end{array}$

Transition State 3d

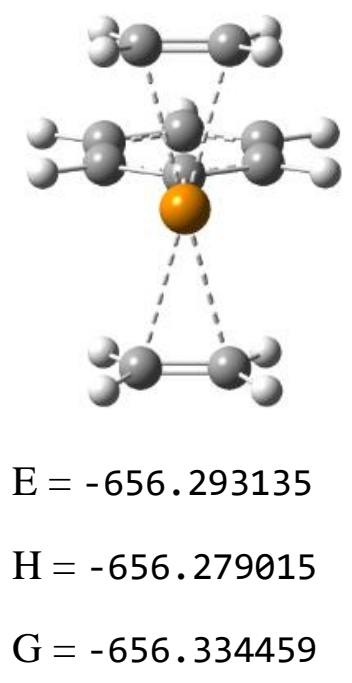

$\mathrm{ZPVE}=0.185826$

Imaginary Frequency $=-127.5900 \mathrm{~cm}^{-1}$

Symbol

X Y

$\mathrm{C}$
$\mathrm{C}$
$\mathrm{H}($ Iso $=2)$
$\mathrm{H}($ Iso $=2)$
$\mathrm{H}($ Iso $=2)$
$\mathrm{H}($ Iso $=2)$
$\mathrm{C}$
$\mathrm{H}$
$\mathrm{H}$
$\mathrm{C}$
$\mathrm{H}$
$\mathrm{H}$
$\mathrm{C}$

\begin{abstract}
1.29877100
1.29890900

0.37156900

0.37131800

2. 21547700

2.21572800

1.29890900

2.21572800

0.37156900

1.29877100

0.37131800

2.21547700

$-0.66060100$
\end{abstract}

1.22401900

$-0.66051800$

$-1.23257000$

$-1.21980800$

$-0.02134800$

1.18833200

$-1.23510300$

$-2.17603800$

1.17495900

$-0.03299200$

$-2.17462300$

0.03756900

$-0.01200400$ 


$\begin{array}{lrrr}\text { C } & -1.34852300 & 0.00001300 & 1.21052600 \\ \text { C } & -1.34830200 & -0.00001300 & -1.21077900 \\ \text { C } & -2.73660900 & 0.00001300 & 1.20418500 \\ \text { H } & -0.81277100 & 0.00002200 & 2.15194500 \\ \text { C } & -2.73639000 & -0.00001300 & -1.20469000 \\ \text { H } & -0.81237800 & -0.00002200 & -2.15210000 \\ \text { C } & -3.42738100 & 0.00000000 & -0.00031600 \\ \text { H } & -3.27633400 & 0.00002200 & 2.14175500 \\ \text { H } & -3.27594300 & -0.00002200 & -2.14235900 \\ \text { H } & -4.50924500 & 0.00000000 & -0.00041400 \\ \text { Te } & 1.44328900 & 0.00000000 & 0.00013300\end{array}$

Dissociation Complex 3e

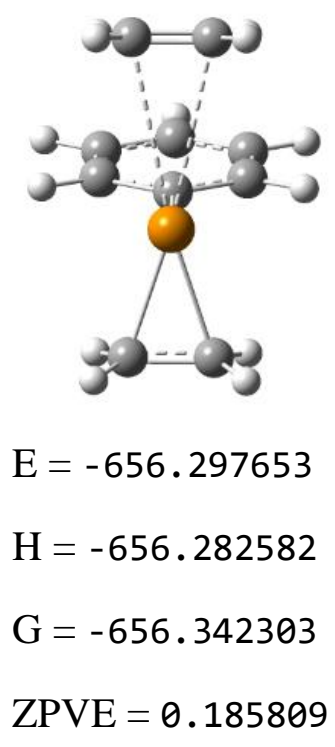

Symbol

X

Y

Z

$\mathrm{C}$
$\mathrm{C}$
$\mathrm{H}$
$\mathrm{H}$
$\mathrm{H}$
$\mathrm{H}$
$\mathrm{C}$
$\mathrm{H}($ IsO $=2)$
$\mathrm{H}($ IsO $=2)$
$\mathrm{C}$
$\mathrm{H}($ IsO $=2)$
$\mathrm{H}($ IsO $=2)$
$\mathrm{C}$
$\mathrm{C}$
$\mathrm{C}$
$\mathrm{C}$
$\mathrm{H}$
$\mathrm{C}$
$\mathrm{H}$
$\mathrm{C}$

$-1.84330300$

$-2.07085000$

$-0.73113800$

0.68140000

$-1.83797300$

.08025900

1.21942800

$-0.95795100$

$-2.38183800$

$-2.36516500$

$-2.78064100$

$-2.07871600$

$-2.77118700$

$-2.09541400$

3.07510000

3.42476300

$-1.38926500$

2.75914300

$-1.27983400$

$-1.27233000$

1.22952100

0.66850500

1.24462800

0.33524800

3.08432400

1.22401900

$-0.55001000$

2.77631400

$-0.66051800$

0.31809400

3.44185700

$-1.23257000$

$-1.40651000$

$-0.17303500$

$-1.21980800$

0.60755400

$-0.21762400$

$-0.02134800$

1.28343300

$-0.23642100$

$-1.23671100$

1.29208800

$-0.34139500$

1.18833200

2.66582200

$-0.15511900$

$-1.23510300$

0.74760600

$-0.36013100$

$-2.17603800$

2.67441200

$-0.18831600$

1.17495900

0.76301500

$-0.41288700$

2.13231400

$-0.03299200$ 


$\begin{array}{lrrr}\mathrm{H} & 3.20061500 & -0.38008900 & -2.17462300 \\ \mathrm{H} & 3.21588500 & -0.41338800 & 2.10993600 \\ \mathrm{H} & 4.43493800 & -0.50789900 & -0.03756900 \\ \mathrm{Te} & -1.47878400 & 0.05674300 & -0.01200400\end{array}$

Telluriranium Product Ion 3f

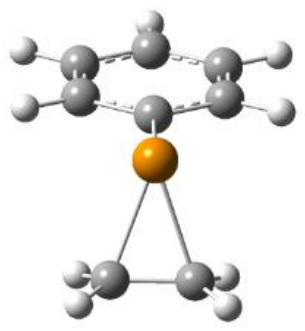

$E=-577.753059$

$H=-577.743222$

$G=-577.789096$

$\mathrm{ZPVE}=0.145781$

Symbol

\section{C}

$\mathrm{H}$

C

$\mathrm{H}$

$\mathrm{H}$

C

C

C

C

$\mathrm{H}$

C

$\mathrm{H}$

C

$\mathrm{H}$

$\mathrm{H}$

$\mathrm{H}$

$\mathrm{H}$

Te

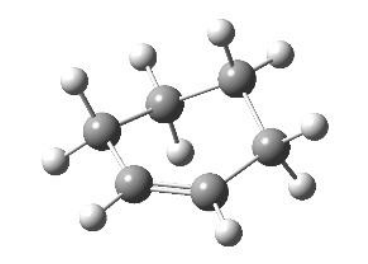

$E=-234.499713$

\section{Cyclohexene-d10}

X

2.30758200

1.52691100

2.48501400

1.83733500

3.12781400

$-0.54338100$

$-1.02289100$

$-1.32353000$

$-2.30232100$

$-0.41737200$

$-2.60071000$

$-0.95107500$

$-3.08586900$

$-2.68656800$

$-3.21670800$

$-4.08409200$

3.43880100

1.36353800
Y

1.13568200

1.62190100

$-0.28116500$

$-0.85788800$

1.75858500

$-0.01125000$

$-1.27493700$

1.13033900

$-1.38841800$

$-2.16110200$

0.99904000

2.10865000

$-0.25532400$

$-2.36391200$

1.87802400

$-0.35112300$

$-0.72465400$

0.16668900
Z

0.63757300

1.20854300

0.74031500

1. 38822400

0.30436800

$-0.27749200$

0.05594100

$-0.11567600$

0.57956700

$-0.08833500$

0.40928700

$-0.39295900$

0.75564000

0.84544000

0.54300000

1.16155400

0.48457500

$-1.12346500$ 
$H=-234.492142$

$G=-234.528780$

$\mathrm{ZPVE}=0.114171$

Symbol

X

Y

Z

$\mathrm{C}$
$\mathrm{C}$
$\mathrm{C}$
$\mathrm{C}$
$\mathrm{C}$
$\mathrm{C}$
$\mathrm{H}(\mathrm{IsO}=2)$
$\mathrm{H}(\mathrm{IsO}=2)$
$\mathrm{H}(\mathrm{IsO}=2)$
$\mathrm{H}($ Iso $=2)$
$\mathrm{H}($ Iso $=2)$
$\mathrm{H}($ Iso $=2)$
$\mathrm{H}($ Iso $=2)$
$\mathrm{H}($ Iso $=2)$
$\mathrm{H}($ Iso $=2)$
$\mathrm{H}($ Iso $=2)$

$$
0.66136200
$$

1.29625600

0.06006100

$-0.66136200$

1.29625600

$-0.06006100$

$-1.48917200$

0.04202400

$-0.11162000$

$-0.68856500$

$-1.18126700$

0.32844500

0.68856500

$-1.18126700$

1.48917200

0.04202400

$-0.32844500$

0.11162000

$-2.37473000$

0.16382700

$-1.18890700$

2.24244800

1.18890700

2. 24244800

$-0.56334000$

$-1.15602300$

$-1.23343600$

$-2.09593100$

1.23343600

$-2.09593100$

0.56334000

1.86459700

$-1.15602300$

$-0.10225600$

0.51698400

$-0.11903300$

0.11903300

1.41533700

0.08968500

$-0.08968500$

$-1.41533700$

2.37473000

0.16382700

1.13116400

$-1.86459700$

$-0.10225600$

$-0.51698400$

$-1.13116400$

\section{Thiiranium Reactant Ion 4b}

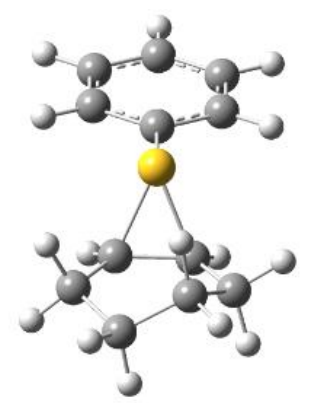

$E=-863.950979$

$H=-863.938542$

$\mathrm{G}=-863.989449$

$\mathrm{ZPVE}=0.242955$

Symbol

$\mathrm{X}$

Y

Z

S
C
H
C
H
C
C
C
0.25667500
1.16749300
0.43180200
1.15027000
0.41757700
$-1.44095500$
$-2.09286700$
$-2.10319200$

0.02396400

0.82808800

$-0.76133400$

$-0.60543100$

$-1.27501800$

$-1.21341300$

0.70603900

$-0.66363400$

1.16151000

$-1.32042000$

0.00656000

0.31255000

1.21495400

0.09832100

$-1.21234900$

0.22910900 


$\begin{array}{lrrr}\mathrm{C} & -3.43929300 & 1.19394200 & -0.23095500 \\ \mathrm{H} & -1.56360700 & 2.15532100 & 0.19049600 \\ \mathrm{C} & -3.44927700 & -1.21524700 & -0.10241100 \\ \mathrm{H} & -1.58171500 & -2.14173000 & 0.42161500 \\ \mathrm{C} & -4.11256500 & -0.01679200 & -0.33237000 \\ \mathrm{H} & -3.96200700 & 2.12471600 & -0.40388700 \\ \mathrm{H} & -3.97962700 & -2.15483100 & -0.17617100 \\ \mathrm{H} & -5.16358700 & -0.02612900 & -0.58817900 \\ \mathrm{C} & 2.43317500 & -1.52536500 & -0.25005600 \\ \mathrm{H} & 2.30114100 & -2.01795900 & 0.71447600 \\ \mathrm{H} & 2.54009000 & -2.32275500 & -0.98608700 \\ \mathrm{C} & 3.68033900 & -0.63861100 & -0.23074500 \\ \mathrm{H} & 4.01319700 & -0.45279600 & -1.25510100 \\ \mathrm{H} & 4.48560400 & -1.17405700 & 0.26962800 \\ \mathrm{C} & 3.41111900 & 0.69859100 & 0.45151100 \\ \mathrm{H} & 3.04923500 & 0.53841600 & 1.47292600 \\ \mathrm{H} & 4.32977100 & 1.27674100 & 0.53785200 \\ \mathrm{C} & 2.39305400 & 1.50030400 & -0.35412900 \\ \mathrm{H} & 2.82407600 & 1.76627600 & -1.32491000 \\ \mathrm{H} & 2.12033900 & 2.43513700 & 0.13557900\end{array}$

Association Complex 4c

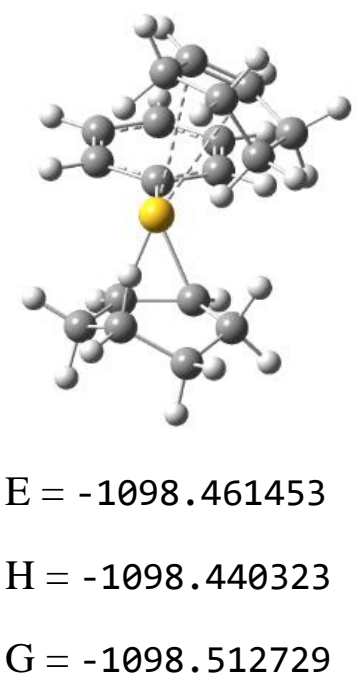

$\mathrm{ZPVE}=0.357724$

Symbol

X

$-1.01524800$

$-0.64294800$

$-1.43769400$

$-2.05702200$

1.43242700

0.61850800

1.72731900

1.12984000

$-1.63492100$

$-2.36715200$

$-1.92190900$
1.19394200

2.15532100

1.21524700

2.14173000

0.01679200

$-1.52536500$

01795900

0.45279600

$-1.17405700$

0.69859100

0.53841600

1.27674100

1.50030400

2.43513700
$-0.23095500$

0.42161500

0.33237000

0.40388700

0.58817900

0.25005600

$-0.23074500$

0.45151100

0.53785200

0.13557900

$\mathrm{H}$ (Iso $=2)$

$\mathrm{H}$ ( Iso $=2)$

C

C

C

2.73142500

2. 54123100

2. 23539600

2.57981800

$-1.46148300$

$-2.01273000$

$-1.68725500$

$-2.39659600$

0.00933000

$-0.34057100$

$-0.51501600$
Z

$-0.75608700$

0.64976300

1.31995600

$-1.01411000$

0.70779500

1.16848900

$-0.56980800$

$-1.13376200$

$-0.22913900$

$-1.35595700$

1.02465000 


\begin{tabular}{|c|c|c|c|}
\hline C & -3.42700300 & -1.22397400 & -1.21375500 \\
\hline $\mathrm{H}$ & -2.11561000 & 0.06565500 & -2.32786000 \\
\hline C & -2.98594300 & -1.39399100 & 1.15131800 \\
\hline $\mathrm{H}$ & -1.32008500 & -0.24906700 & 1.88534500 \\
\hline C & -3.73527200 & -1.74585500 & 0.03532400 \\
\hline $\mathrm{H}$ & -4.00928900 & -1.50440700 & -2.08084400 \\
\hline $\mathrm{H}$ & -3.22587800 & -1.80795100 & 2.12124000 \\
\hline $\mathrm{H}$ & -4.56276600 & -2.43461900 & 0.14009900 \\
\hline C & 2.18945700 & -0.49615700 & 1.57794900 \\
\hline $\mathrm{H}(\mathrm{IsO}=2)$ & 1.48133300 & 0.13525100 & 2.13027500 \\
\hline $\mathrm{H}(\mathrm{IsO}=2)$ & 2.72810400 & -1.05743300 & 2.34822700 \\
\hline $\mathrm{C}$ & 2.89211300 & -1.04508100 & -1.27050400 \\
\hline $\mathrm{H}(\mathrm{IsO}=2)$ & 2.53170000 & -0.25593700 & -1.94361000 \\
\hline $\mathrm{H}(\mathrm{IsO}=2)$ & 3.37547100 & -1.78151600 & -1.91514400 \\
\hline C & 3.16746300 & 0.36629400 & 0.77928800 \\
\hline $\mathrm{H}(\mathrm{IsO}=2)$ & 2.62316600 & 1.16727900 & 0.26476800 \\
\hline $\mathrm{H}(\mathrm{IsO}=2)$ & 3.87625400 & 0.85094000 & 1.45198600 \\
\hline $\mathrm{C}$ & 3.89250600 & -0.46675700 & -0.27321100 \\
\hline $\mathrm{H}(\mathrm{IsO}=2)$ & 4.64216600 & 0.13281600 & -0.78991200 \\
\hline $\mathrm{H}(\mathrm{IsO}=2)$ & 4.42079200 & -1.28663300 & 0.22043400 \\
\hline C & 0.53934600 & 3.26641900 & 1.26908900 \\
\hline $\mathrm{H}$ & 0.18017600 & 3.72597300 & 2.19076700 \\
\hline $\mathrm{H}$ & 1.29184500 & 2.53475600 & 1.56872800 \\
\hline C & -0.20280600 & 3.64186300 & -1.64144800 \\
\hline $\mathrm{H}$ & -0.73696600 & 4.59720500 & -1.66567400 \\
\hline $\mathrm{H}$ & -0.19991400 & 3.25588600 & -2.66086700 \\
\hline C & 1.15075100 & 4.32608700 & 0.34758700 \\
\hline $\mathrm{H}$ & 0.55404100 & 5.24026800 & 0.39863300 \\
\hline $\mathrm{H}$ & 2.14521100 & 4.57931700 & 0.71206300 \\
\hline C & 1.20858900 & 3.86206000 & -1.10450900 \\
\hline $\mathrm{H}$ & 1.71234800 & 4.60498600 & -1.72107700 \\
\hline $\mathrm{H}$ & 1.79619400 & 2.94106900 & -1.18897700 \\
\hline$S$ & -0.22403500 & 1.06832900 & -0.42866000 \\
\hline
\end{tabular}

Transition State 4d

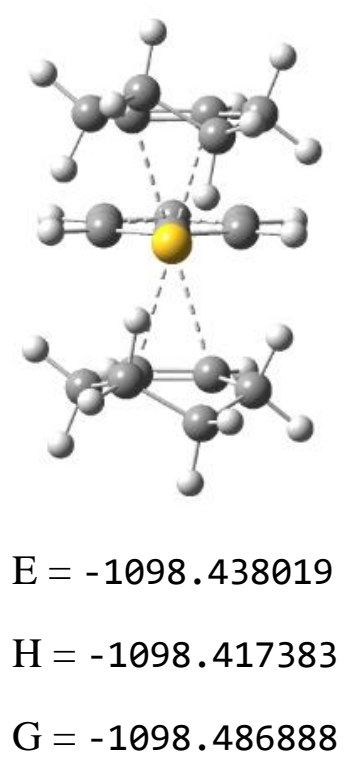




\begin{tabular}{|c|c|c|c|}
\hline \multicolumn{4}{|c|}{ Imaginary Frequency $=-270.3913 \mathrm{~cm}^{-1}$} \\
\hline Symbol & $X$ & $\mathrm{Y}$ & $\mathrm{Z}$ \\
\hline C & -0.98944900 & 2.72799000 & -0.75936500 \\
\hline C & -0.90644700 & 2.81590700 & 0.60175500 \\
\hline $\mathrm{H}(\mathrm{IsO}=2)$ & -1.70357400 & 2.38804800 & 1.19987700 \\
\hline $\mathrm{H}(\mathrm{ISO}=2)$ & -1.86233400 & 2.25909600 & -1.20008900 \\
\hline $\mathrm{C}$ & 1.33360200 & -1.20365800 & 0.68279400 \\
\hline $\mathrm{H}$ & 0.51127200 & -1.72004900 & 1.16558500 \\
\hline C & 1.40057100 & -1.20464800 & -0.68198300 \\
\hline $\mathrm{H}$ & 0.61275000 & -1.69793000 & -1.24073200 \\
\hline C & -1.42123500 & -0.16493500 & -0.00035100 \\
\hline C & -2.01689600 & -0.56399500 & -1.19443900 \\
\hline C & -2.02036700 & -0.46097800 & 1.22164100 \\
\hline C & -3.21444100 & -1.26150500 & -1.16252800 \\
\hline $\mathrm{H}$ & -1.54368800 & -0.32600100 & -2.13960800 \\
\hline C & -3.21892600 & -1.15710400 & 1.24557500 \\
\hline $\mathrm{H}$ & -1.54684600 & -0.14848500 & 2.14469600 \\
\hline C & -3.81398800 & -1.55672700 & 0.05548800 \\
\hline $\mathrm{H}$ & -3.67965600 & -1.57354700 & -2.08785200 \\
\hline $\mathrm{H}$ & -3.68748400 & -1.38822600 & 2.19270700 \\
\hline $\mathrm{H}$ & -4.74896100 & -2.10052900 & 0.07742300 \\
\hline C & 2.43454000 & -0.67616400 & 1.54838800 \\
\hline $\mathrm{H}$ & 2.00729800 & -0.12504900 & 2.38905500 \\
\hline $\mathrm{H}$ & 2.94038200 & -1.54464600 & 1.98643700 \\
\hline C & 2.59589300 & -0.70486700 & -1.43780800 \\
\hline $\mathrm{H}$ & 2.33402300 & 0.23651500 & -1.93432000 \\
\hline $\mathrm{H}$ & 2.80720100 & -1.41197300 & -2.24261400 \\
\hline C & 3.43151600 & 0.17476900 & 0.76567300 \\
\hline $\mathrm{H}$ & 2.98630800 & 1.15066800 & 0.54454000 \\
\hline $\mathrm{H}$ & 4.31201900 & 0.36505600 & 1.37812300 \\
\hline C & 3.81727100 & -0.51700800 & -0.53798300 \\
\hline $\mathrm{H}$ & 4.58032500 & 0.05195700 & -1.06769000 \\
\hline $\mathrm{H}$ & 4.25099500 & -1.49400000 & -0.30956700 \\
\hline C & 0.14641100 & 3.62320000 & 1.30174900 \\
\hline $\mathrm{H}(\mathrm{IsO}=2)$ & -0.33379000 & 4.17453500 & 2.11279900 \\
\hline $\mathrm{H}(\mathrm{IsO}=2)$ & 0.85297900 & 2.94060000 & 1.78772700 \\
\hline C & -0.01909900 & 3.40411900 & -1.67641100 \\
\hline $\mathrm{H}(\mathrm{IsO}=2)$ & -0.53911200 & 4.26464900 & -2.11356500 \\
\hline $\mathrm{H}(\mathrm{IsO}=2)$ & 0.21657800 & 2.74222400 & -2.51269100 \\
\hline $\mathrm{C}$ & 0.88009000 & 4.57104000 & 0.35311800 \\
\hline $\mathrm{H}(\mathrm{IsO}=2)$ & 0.23762200 & 5.42659000 & 0.12931500 \\
\hline $\mathrm{H}(\mathrm{IsO}=2)$ & 1.77104400 & 4.96355300 & 0.84163900 \\
\hline C & 1.24188700 & 3.86434800 & -0.94944000 \\
\hline $\mathrm{H}(\mathrm{IsO}=2)$ & 1.82095900 & 4.52050800 & -1.59804800 \\
\hline $\mathrm{H}(\mathrm{IsO}=2)$ & 1.87611000 & 2.99830700 & -0.73206300 \\
\hline $\mathrm{s}$ & 0.11188600 & 0.72730700 & -0.03652000 \\
\hline
\end{tabular}


Dissociation Complex 4e

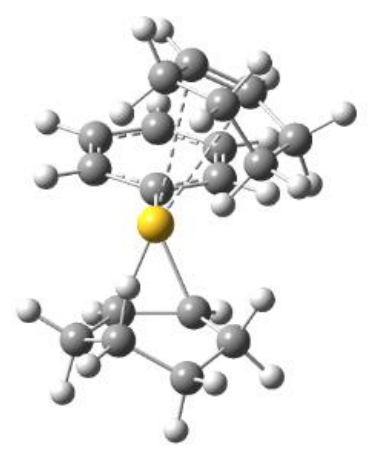

$E=-1098.462051$

$H=-1098.440943$

$\mathrm{G}=-1098.513189$

$\mathrm{ZPVE}=0.357126$

Symbol

$$
\text { X }
$$

Y

Z

$\mathrm{C}$
$\mathrm{C}$
$\mathrm{H}($ IsO $=2)$
$\mathrm{H}($ IsO $=2)$
$\mathrm{C}$
$\mathrm{H}$
$\mathrm{C}$
$\mathrm{H}$
$\mathrm{C}$
$\mathrm{C}$
$\mathrm{C}$
$\mathrm{C}$
$\mathrm{H}$
$\mathrm{C}$
$\mathrm{H}$
$\mathrm{C}$
$\mathrm{H}$
$\mathrm{H}$
$\mathrm{H}$
$\mathrm{C}$
$\mathrm{H}$
$\mathrm{H}$
$\mathrm{C}$
$\mathrm{H}$
$\mathrm{H}$
$\mathrm{C}$
$\mathrm{H}$
$\mathrm{H}$
$\mathrm{C}$
$\mathrm{H}$
$\mathrm{H}$
$\mathrm{C}$

$-1.01563500$

$-0.64330600$

$-1.43800000$

$-2.05737400$

1.43292600

0.61911600

1.72764700

1.13013600

$-1.63472500$

$-2.36702600$

$-1.92145300$

$-3.42669000$

$-2.11567400$

$-2.98531200$

$-1.31957400$

$-3.73471100$

$-4.00903300$

$-3.22504500$

$-4.56206900$

2.19001100

1.48193200

2.72885500

2.89227400

2.53169200

3.37562600

3.16779700

2.62332000

3.87665400

3.89275300

4.64227200

4.42120600

0.53882800
2.73096500

2.54112700

2. 23528300

2.57904900

$-1.46119700$

$-2.01255000$

$-1.68682500$

$-2.39615600$

0.00886200

$-0.34120500$

$-0.51549200$

$-1.22479900$

0.06503800

$-1.39466600$

$-0.24940800$

$-1.74669800$

$-1.50537100$

$-1.80863500$

$-2.43560900$

$-0.49591200$

0.13536200

$-1.05723900$

$-1.04447100$

$-0.25531200$

$-1.78080300$

0.36673300

1.16772800

0.85136300

$-0.46612300$

0.13357200

$-1.28598700$

3.26671100
$-0.75641200$

0.64948000

1.31973300

$-1.01439200$

0.70811000

1.16887300

$-0.56956000$

$-1.13349100$

$-0.22899100$

$-1.35571200$

1.02484800

$-1.21334800$

$-2.32765800$

1.15167800

1.88546200

0.03579000

$-2.08035400$

2.12164500

0.14068000

1.57826500

2.13078700

2. 34836600

$-1.27036700$

$-1.94337100$

$-1.91513300$

0.77953500

0.26521700

1.45217200

$-0.27317300$

$-0.78993800$

0.22031400

1.26866800 


\begin{tabular}{|c|c|c|c|}
\hline $\mathrm{H}(\mathrm{IsO}=2)$ & 0.17960000 & 3.72621100 & 2.19034900 \\
\hline $\mathrm{H}(\mathrm{IsO}=2)$ & 1.29158100 & 2.53529800 & 1.56827900 \\
\hline C & -0.20346500 & 3.64151700 & -1.64190600 \\
\hline $\mathrm{H}(\mathrm{ISO}=2)$ & -0.73794800 & 4.59667700 & -1.66625900 \\
\hline $\mathrm{H}(\mathrm{IsO}=2)$ & -0.20042600 & 3.25540600 & -2.66127100 \\
\hline C & 1.14986100 & 4.32647700 & 0.34703700 \\
\hline $\mathrm{H}(\mathrm{IsO}=2)$ & 0.55283300 & 5.24045900 & 0.39797000 \\
\hline $\mathrm{H}(\mathrm{IsO}=2)$ & 2.14423000 & 4.58010100 & 0.71148700 \\
\hline C & 1.20786000 & 3.86227200 & -1.10498900 \\
\hline $\mathrm{H}(\mathrm{IsO}=2)$ & 1.71136300 & 4.60527300 & -1.72167300 \\
\hline $\mathrm{H}(\mathrm{IsO}=2)$ & 1.79578600 & 2.94147100 & -1.18934500 \\
\hline $\mathrm{s}$ & -0.22405500 & 1.06813900 & -0.4286800 \\
\hline
\end{tabular}

\section{Thiiranium Product Ion $4 f$}

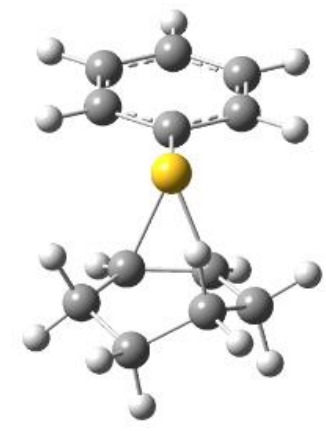

$E=-863.984801$

$H=-863.971181$

$\mathrm{G}=-864.024272$

$\mathrm{ZPVE}=0.209132$

Symbol

X

Y

Z

$\mathrm{C}$
$\mathrm{H}($ Iso $=2)$
$\mathrm{C}$
$\mathrm{H}($ Iso $=2)$
$\mathrm{C}$
$\mathrm{C}$
$\mathrm{C}$
$\mathrm{C}$
$\mathrm{H}$
$\mathrm{C}$
$\mathrm{H}$
$\mathrm{C}$
$\mathrm{H}$
$\mathrm{H}$
$\mathrm{H}$
$\mathrm{C}$
$\mathrm{H}($ Iso $=2)$
$\mathrm{H}($ Iso $=2)$
$\mathrm{C}$

1.13238000

0.77582000

0.78658600

0.39268700

1.29751300

1.38278300

1.11444900

$-0.69061300$

0.86358900

0.37724900

$-1.13760500$

1.52114200

$-1.46986700$

$-0.00362800$

$-2.12323900$

$-1.20926400$

$-0.13813200$

$-2.13215900$

1.21634200

0.08678700

$-3.47157700$

$-1.18401900$

$-0.07328600$

$-2.15075200$

0.40797400

$-1.59369600$

1. 22346200

0.00896200

$-3.48017100$

2.14322400

0.25039500

$-1.60934600$

0.02798300

$-0.27398700$

$-4.14506800$

$-2.11251900$

0.49095600

$-3.99556100$

0.58910800

$-4.01072000$

2.16394700

0.30982300

$-5.19753600$

0.04062700

0.74060100

2.40054900

1.53507900

0.42993500

2.27514300

2.01515100

$-0.54176200$

2.50259300

2. 34189900

1.15631300

3.64766800

0.64803400

0.43059200 


$\begin{array}{lrrr}\text { H }(\text { Iso }=2) & 3.97349700 & 0.47547500 & 1.45951500 \\ \text { H(Iso }=2) & 4.45641400 & 1.17688300 & -0.07117200 \\ \mathrm{C} & 3.38283800 & -0.69783700 & -0.23615300 \\ \mathrm{H}(\text { Iso }=2) & 3.02802700 & -0.55081600 & -1.26202200 \\ \mathrm{H}(\text { Iso }=2) & 4.30191600 & -1.27719400 & -0.30869700 \\ \mathrm{C} & 2.35907800 & -1.48897300 & 0.57272300 \\ \mathrm{H}(\text { Iso }=2) & 2.78335100 & -1.74258700 & 1.54977300 \\ \mathrm{H}(\text { Iso }=2) & 2.08949400 & -2.42992500 & 0.09311900 \\ \mathrm{~S} & 0.23093300 & -0.02773400 & -0.64278000\end{array}$

Cyclohexene

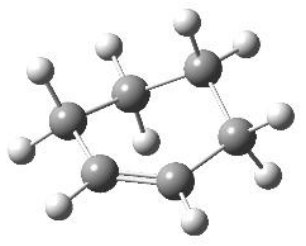

$E=-234.466555$

$H=-234.460147$

$G=-234.495133$

$\mathrm{ZPVE}=0.147371$

Symbol

$\mathrm{X}$

Y

Z

C

0.66136200

1.29625600

0.06006100

$-0.66136200$

1.29625600

$-0.06006100$

C

$-1.48917200$

0.04202400

$-0.11162000$

$-0.68856500$

$-1.18126700$

0.32844500

0.68856500

$-1.18126700$

$-0.32844500$

1.48917200

0.04202400

0.11162000

$-2.37473000$

0.16382700

0.51698400

$-1.18890700$

2.24244800

$-0.11903300$

1.18890700

2.24244800

0.11903300

$-0.56334000$

$-1.15602300$

1.41533700

$-1.23343600$

$-2.09593100$

0.08968500

1.23343600

$-2.09593100$

$-0.08968500$

0.56334000

$-1.15602300$

$-1.41533700$

1.86459700

$-0.10225600$

1.13116400

2.37473000

0.16382700

$-0.51698400$

$-1.86459700$

$-0.10225600$

$-1.13116400$ 
Seleniranium Reactant Ion 5b

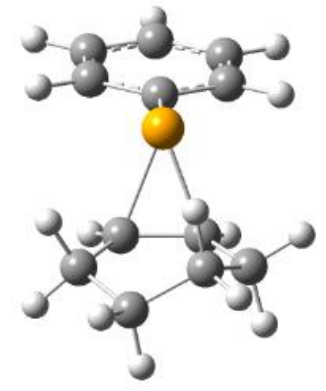

$E=-2867.342687$

$H=-2867.329728$

$\mathrm{G}=-2867.382414$

$\mathrm{ZPVE}=0.241512$

Symbol

$$
\text { X }
$$

Y

Z

$\mathrm{C}$
$\mathrm{H}$
$\mathrm{C}$
$\mathrm{H}$
$\mathrm{C}$
$\mathrm{C}$
$\mathrm{C}$
$\mathrm{C}$
$\mathrm{H}$
$\mathrm{C}$
$\mathrm{H}$
$\mathrm{C}$
$\mathrm{H}$
$\mathrm{H}$
$\mathrm{H}$
$\mathrm{C}$
$\mathrm{H}$
$\mathrm{H}$
$\mathrm{C}$
$\mathrm{H}$
$\mathrm{H}$
$\mathrm{C}$
$\mathrm{H}$
$\mathrm{H}$
$\mathrm{C}$
$\mathrm{H}$
$\mathrm{H}$
$\mathrm{S}$

\subsection{0}

0.76818800

0.74836900

0.40441300

1.29349800

1.29007500

1.16160300

$-0.68259200$

0.82442700

0.38421100

$-1.13397500$

1.43129400

$-1.57634600$

$-0.00769000$

$-0.23814200$

$-2.21492900$

$-1.21046300$

0.03886000

$-2.23193100$

1. 21308800

$-0.13351500$

$-3.53891000$

$-1.18170200$

$-1.69539500$

$-2.15448600$

0.45084500

$-3.55576100$

1.22422800

$-0.06826100$

$-1.72531700$

2.13954400

0.28026000

$-4.20440200$

0.03140500

$-0.37314800$

$-4.05040000$

$-2.10864100$

0.57202100

$-4.08022900$

2.16592800

0.67201200

$-5.23783100$

0.04682700

0.36923800

2.46066200

1.53274000

0.89140200

2.37000900

2.04550900

0.45208400

2.53677100

2. 31906700

$-0.50632500$

3.70902000

0.64642800

1.20474600

4.01507500

0.47156100

0.47260100

4.52727400

1.17663300

1.50709400

3.46008400

$-0.70019000$

$-0.01186900$

3.13455300

$-0.55564300$

$-0.19893200$

4.37993700

$-1.28099300$

$-1.23541100$

2. 41100000

$-1.48450200$

$-0.24509400$

2.80301200

$-1.72708600$

0.58211600

2.15990900

$-2.43261400$

1.57656700

0.10735700

0.23029000

$-0.03609500$

$-0.87798700$ 


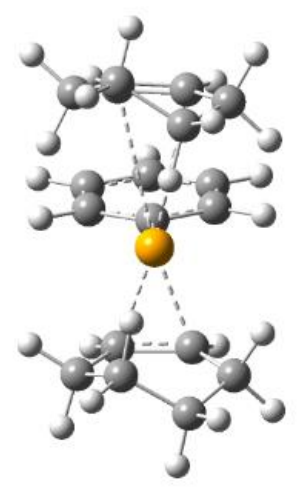

$E=-3101.854613$

$H=-3101.832798$

$\mathrm{G}=-3101.908173$

$\mathrm{ZPVE}=0.356074$

Symbol

X

Y

Z

\begin{tabular}{|c|c|c|c|}
\hline C & -0.90663300 & 2.76787400 & -0.83980300 \\
\hline C & -0.91516200 & 2.85009800 & 0.59992000 \\
\hline $\mathrm{H}$ & -1.81415500 & 2.51384300 & 1.103498 \\
\hline $\mathrm{H}$ & -1.80281700 & 2.39400600 & -1.322353 \\
\hline C & 1.54260500 & -1.53654700 & 0.756430 \\
\hline $\mathrm{H}(\mathrm{IsO}=2)$ & 0.72607300 & -1.97319400 & 1.324617 \\
\hline C & 1.55510700 & -1.67093400 & -0.569898 \\
\hline $\mathrm{H}(\mathrm{IsO}=2)$ & 0.73579500 & -2.19319900 & -1.055458 \\
\hline C & -1.53127900 & -0.04164300 & -0.004965 \\
\hline C & -2.03352400 & -0.51899900 & -1.208616 \\
\hline C & -2.07200900 & -0.42555500 & 1.2151540 \\
\hline C & -3.11545900 & -1.38720900 & -1.1848256 \\
\hline $\mathrm{H}$ & -1.58609400 & -0.22662800 & -2.150608 \\
\hline C & -3.15435600 & -1.29375900 & 1.2237536 \\
\hline $\mathrm{H}$ & -1.65463100 & -0.06100700 & $2.145712 e$ \\
\hline C & -3.67380800 & -1.77094700 & $0.027507 €$ \\
\hline $\mathrm{H}$ & -3.51839500 & -1.76581500 & $-2.114484 \ell$ \\
\hline $\mathrm{H}$ & -3.58779900 & -1.59941500 & $2.166424 \ell$ \\
\hline $\mathrm{H}$ & -4.51628300 & -2.44939600 & 0.040212 \\
\hline C & 2.62309100 & -0.83320800 & 1.5293426 \\
\hline $\mathrm{H}(\mathrm{ISO}=2)$ & 2.17082800 & -0.17206200 & 2.275869 \\
\hline $\mathrm{H}(\mathrm{ISO}=2)$ & 3.18511600 & -1.57664500 & 2.104104 \\
\hline C & 2.67885500 & -1.17715200 & -1.437787 \\
\hline $\mathrm{H}(\mathrm{IsO}=2)$ & 2.35636300 & -0.28120700 & -1.985475 \\
\hline $\mathrm{H}(\mathrm{IsO}=2)$ & 2.89641300 & -1.92438600 & -2.203524 \\
\hline C & 3.56727600 & -0.04956900 & 0.618403 \\
\hline $\mathrm{H}(\mathrm{IsO}=2)$ & 3.08229800 & 0.87999600 & 0.297020 \\
\hline $\mathrm{H}(\mathrm{IsO}=2)$ & 4.46249700 & 0.24090800 & 1.1684116 \\
\hline C & 3.92868800 & -0.86729300 & -0.617747 \\
\hline $\mathrm{H}(\mathrm{ISO}=2)$ & 4.66165100 & -0.34114700 & -1.229454 \\
\hline $\mathrm{H}(\mathrm{IsO}=2)$ & 4.39015100 & -1.80655900 & -0.301503 \\
\hline
\end{tabular}




$\begin{array}{lrrr}\text { Se } & 0.01004800 & 1.10122600 & -0.02290500 \\ \text { C } & -0.01476800 & 3.81343000 & 1.35128700 \\ \text { H } & -0.64457800 & 4.33873900 & 2.07105500 \\ \text { H } & 0.71195200 & 3.25395300 & 1.94250900 \\ \text { C } & 0.02203100 & 3.62178000 & -1.65914500 \\ \text { H } & -0.58029400 & 4.46836500 & -2.00823700 \\ \text { H } & 0.34009200 & 3.07906900 & -2.54907800 \\ \text { C } & 0.69569300 & 4.80975200 & 0.43152500 \\ \text { H } & 0.00365100 & 5.60986000 & 0.15679900 \\ \text { H } & 1.51469600 & 5.27572600 & 0.97714100 \\ \text { C } & 1.20098300 & 4.14068500 & -0.84263800 \\ \text { H } & 1.77319100 & 4.84480200 & -1.44478300 \\ \text { H } & 1.88312500 & 3.31871000 & -0.59584700\end{array}$

Transition State 5d

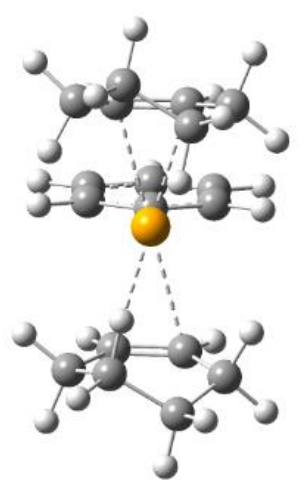

$E=-3101.845980$

$H=-3101.824942$

$\mathrm{G}=-3101.896321$

$\mathrm{ZPVE}=0.355568$

Imaginary Frequency $=-157.5179 \mathrm{~cm}^{-1}$

Symbol

$\mathrm{C}$
$\mathrm{C}$
$\mathrm{H}($ IsO $=2)$
$\mathrm{H}($ IsO $=2)$
$\mathrm{C}$
$\mathrm{H}$
$\mathrm{C}$
$\mathrm{H}$
$\mathrm{C}$
$\mathrm{C}$
$\mathrm{C}$
$\mathrm{C}$
$\mathrm{H}$
$\mathrm{C}$
$\mathrm{H}$

X

$$
\begin{array}{r}
-0.99948400 \\
-0.95767200 \\
-1.74332400 \\
-1.83123000 \\
1.41136100 \\
0.56589200 \\
1.45940800 \\
0.63481700 \\
-1.44480500 \\
-2.03721900 \\
-2.04078900 \\
-3.23654300 \\
-1.56636500 \\
-3.24063900 \\
-1.57104300
\end{array}
$$

Y

$$
\begin{array}{rr}
2.82117200 & -0.78586200 \\
2.91038300 & 0.57682400 \\
2.43200700 & 1.15220800 \\
2.30097300 & -1.24933000 \\
-1.25868100 & 0.70825100 \\
-1.71355500 & 1.21374100 \\
-1.29781700 & -0.65653300 \\
-1.75712700 & -1.19156600 \\
-0.17945000 & -0.00040200 \\
-0.57650700 & -1.19498800 \\
-0.47272600 & 1.22205400 \\
-1.27320800 & -1.16189500 \\
-0.34232400 & -2.14202100 \\
-1.16894200 & 1.24511900 \\
-0.16095200 & 2.14701000
\end{array}
$$




\begin{tabular}{|c|c|c|c|}
\hline C & -3.83620200 & -1.56797000 & 0.05563100 \\
\hline $\mathrm{H}$ & -3.70180900 & -1.58477900 & -2.08745700 \\
\hline $\mathrm{H}$ & -3.70904900 & -1.39946000 & 2.19257400 \\
\hline $\mathrm{H}$ & -4.77164600 & -2.11092300 & 0.07765900 \\
\hline C & 2.56095600 & -0.78697200 & 1.54568600 \\
\hline $\mathrm{H}$ & 2.18833700 & -0.19583700 & 2.38446900 \\
\hline $\mathrm{H}$ & 3.02135200 & -1.67826600 & 1.98768900 \\
\hline C & 2.67935000 & -0.90827200 & -1.44201800 \\
\hline $\mathrm{H}$ & 2.48073000 & 0.02554200 & -1.97851200 \\
\hline $\mathrm{H}$ & 2.83392000 & -1.66312900 & -2.21587400 \\
\hline C & 3.59219700 & -0.01593000 & 0.72606600 \\
\hline $\mathrm{H}$ & 3.19978300 & 0.97720300 & 0.48003600 \\
\hline $\mathrm{H}$ & 4.49047500 & 0.14299400 & 1.32133500 \\
\hline C & 3.91977600 & -0.76874500 & -0.55957900 \\
\hline $\mathrm{H}$ & 4.71041500 & -0.26480200 & -1.11398000 \\
\hline $\mathrm{H}$ & 4.29498500 & -1.76308400 & -0.30400100 \\
\hline Se & 0.21445900 & 0.78578200 & -0.03986600 \\
\hline C & 0.01284200 & 3.79412500 & 1.30775700 \\
\hline $\mathrm{H}(\mathrm{IsO}=2)$ & -0.53737000 & 4.31799400 & 2.09224000 \\
\hline $\mathrm{H}(\mathrm{IsO}=2)$ & 0.74637400 & 3.17086300 & 1.83001300 \\
\hline C & -0.05439900 & 3.56992200 & -1.67561000 \\
\hline $\mathrm{H}(\mathrm{ISO}=2)$ & -0.61859500 & 4.40211000 & -2.11251100 \\
\hline $\mathrm{H}(\mathrm{ISO}=2)$ & 0.24275500 & 2.93730100 & -2.51422500 \\
\hline $\mathrm{C}$ & 0.71270900 & 4.78449800 & 0.37726800 \\
\hline $\mathrm{H}(\mathrm{IsO}=2)$ & 0.02418100 & 5.59688900 & 0.13078000 \\
\hline $\mathrm{H}(\mathrm{IsO}=2)$ & 1.56192200 & 5.23389600 & 0.89040200 \\
\hline C & 1.15603200 & 4.10098300 & -0.91240900 \\
\hline $\mathrm{H}(\mathrm{IsO}=2)$ & 1.71509700 & 4.79047700 & -1.54370300 \\
\hline $\mathrm{H}(\mathrm{IsO}=2)$ & 1.83428000 & 3.27368900 & -0.67491100 \\
\hline
\end{tabular}

Dissociation Complex 5e

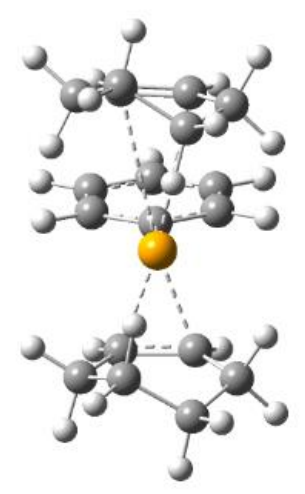

$E=-3101.855123$

$H=-3101.833323$

$G=-3101.908516$

$\mathrm{ZPVE}=0.355563$

Symbol

X

Y

Z 


\begin{tabular}{|c|c|c|c|}
\hline C & -0.90392100 & 2.76541700 & -0.83974600 \\
\hline C & -0.91751700 & 2.85174900 & 0.59972000 \\
\hline $\mathrm{H}(\mathrm{IsO}=2)$ & -1.81825100 & 2.51679000 & 1.10103700 \\
\hline $\mathrm{H}(\mathrm{ISO}=2)$ & -1.79825800 & 2.38965400 & -1.32426900 \\
\hline C & 1.54367800 & -1.53970700 & 0.75136300 \\
\hline $\mathrm{H}$ & 0.72752000 & -1.97826300 & 1.31861500 \\
\hline C & 1.55504900 & -1.66914700 & -0.57545500 \\
\hline $\mathrm{H}$ & 0.73554600 & -2.18993100 & -1.06228100 \\
\hline C & -1.53136000 & -0.04128400 & 0.00091600 \\
\hline C & -2.02820500 & -0.52363900 & -1.20301500 \\
\hline C & -2.07780700 & -0.41988200 & 1.22013100 \\
\hline C & -3.11049500 & -1.39137600 & -1.18056400 \\
\hline $\mathrm{H}$ & -1.57616300 & -0.23539900 & -2.14408800 \\
\hline C & -3.16046400 & -1.28775600 & 1.22740200 \\
\hline $\mathrm{H}$ & -1.66473300 & -0.05146300 & 2.15107800 \\
\hline C & -3.67455900 & -1.76982300 & 0.03082800 \\
\hline $\mathrm{H}$ & -3.50929900 & -1.77378500 & -2.11045100 \\
\hline $\mathrm{H}$ & -3.59833400 & -1.58929600 & 2.16935200 \\
\hline $\mathrm{H}$ & -4.51731200 & -2.44794300 & 0.04251200 \\
\hline C & 2.62509900 & -0.83962200 & 1.52598400 \\
\hline $\mathrm{H}$ & 2.17386100 & -0.18156300 & 2.27580700 \\
\hline $\mathrm{H}$ & 3.18765300 & -1.58561500 & 2.09692000 \\
\hline C & 2.67788100 & -1.17183800 & -1.44251200 \\
\hline $\mathrm{H}$ & 2.35467200 & -0.27365600 & -1.98615400 \\
\hline $\mathrm{H}$ & 2.89469800 & -1.91590000 & -2.21154100 \\
\hline C & 3.56840600 & -0.05240800 & 0.61723200 \\
\hline $\mathrm{H}$ & 3.08314400 & 0.87845400 & 0.30008500 \\
\hline $\mathrm{H}$ & 4.46419600 & 0.23583300 & 1.16748900 \\
\hline C & 3.92854500 & -0.86519100 & -0.62253900 \\
\hline $\mathrm{H}$ & 4.66088400 & -0.33661000 & -1.23289700 \\
\hline $\mathrm{H}$ & 4.39034100 & -1.80570600 & -0.31052000 \\
\hline Se & 0.01015600 & 1.10137600 & -0.01510000 \\
\hline C & -0.02008300 & 3.81760900 & 1.35138400 \\
\hline $\mathrm{H}(\mathrm{IsO}=2)$ & -0.65250800 & 4.34462900 & 2.06759700 \\
\hline $\mathrm{H}(\mathrm{IsO}=2)$ & 0.70497400 & 3.26019900 & 1.94656900 \\
\hline C & 0.02700800 & 3.61748800 & -1.65836000 \\
\hline $\mathrm{H}(\mathrm{IsO}=2)$ & -0.57469200 & 4.46274900 & -2.01175400 \\
\hline $\mathrm{H}(\mathrm{IsO}=2)$ & 0.34831100 & 3.07252700 & -2.54575600 \\
\hline C & 0.69295200 & 4.81171200 & 0.43119600 \\
\hline $\mathrm{H}(\mathrm{IsO}=2)$ & 0.00141900 & 5.61066500 & 0.15186100 \\
\hline $\mathrm{H}(\mathrm{IsO}=2)$ & 1.50985200 & 5.27967700 & 0.97826000 \\
\hline C & 1.20291800 & 4.13930100 & -0.83933800 \\
\hline $\mathrm{H}(\mathrm{IsO}=2)$ & 1.77683400 & 4.84197800 & -1.44153900 \\
\hline $\mathrm{H}(\mathrm{IsO}=2)$ & 1.88458800 & 3.31834500 & -0.58789800 \\
\hline
\end{tabular}


Seleniranium Product Ion 5f

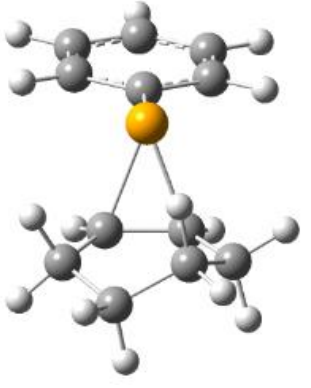

$E=-2867.376449$

$H=-2867.362295$

$\mathrm{G}=-2867.417162$

$\mathrm{ZPVE}=0.207750$

Symbol

X

Y

Z

$\mathrm{C}$

$\mathrm{H}(\mathrm{IsO}=2)$

C

$\mathrm{H}(\mathrm{IsO}=2)$

C

C

C

$\mathrm{C}$

$\mathrm{H}$

C

$\mathrm{H}$

C

$\mathrm{H}$

$\mathrm{H}$

$\mathrm{H}$

C

$\mathrm{H}$ ( Iso $=2)$

$\mathrm{H}(\mathrm{IsO}=2)$

$\mathrm{C}$

$\mathrm{H}$ ( Iso $=2)$

$\mathrm{H}(\mathrm{IsO}=2)$

C

$\mathrm{H}(\mathrm{ISO}=2)$

$\mathrm{H}(\mathrm{ISO}=2)$

C

$\mathrm{H}$ ( Iso $=2)$

$\mathrm{H}$ (IsO $=2$ )

$\mathrm{Se}$

\subsection{0}

0.76818800

0.74836900

0.40441300

1.29349800

1.29007500

1.16160300

$-0.68259200$

0.82442700

0.38421100

$-1.13397500$

1.43129400

$-1.57634600$

$-0.00769000$

$-0.23814200$

$-2.21492900$

$-1.21046300$

0.03886000

$-2.23193100$

1. 21308800

$-0.13351500$

$-3.53891000$

$-1.18170200$

$-1.69539500$

$-2.15448600$

0.45084500

$-3.55576100$

1.22422800

$-0.06826100$

$-1.72531700$

2.13954400

0.28026000

$-4.20440200$

0.03140500

$-0.37314800$

$-4.05040000$

$-2.10864100$

0.57202100

$-4.08022900$

2.16592800

0.67201200

$-5.23783100$

0.04682700

0.36923800

2.46066200

1.53274000

0.89140200

2.37000900

2.04550900

0.45208400

2.53677100

2. 31906700

$-0.50632500$

3.70902000

0.64642800

1.20474600

4.01507500

0.47156100

0.47260100

4.52727400

1.17663300

1.50709400

3.46008400

$-0.70019000$

$-0.01186900$

3.13455300

$-0.55564300$

$-0.19893200$

4.37993700

$-1.28099300$

$-1.23541100$

2.41100000

$-1.48450200$

$-0.24509400$

2.80301200

$-1.72708600$

0.58211600

2.15990900

$-2.43261400$

1.57656700

0.23029000

$-0.03609500$

0.10735700

$-0.87798700$ 
Telluriranium Reactant Ion $6 \mathrm{~b}$

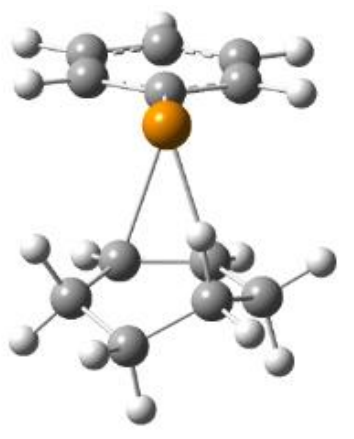

$E=-733.708124$

$H=-733.694756$

$\mathrm{G}=-733.749114$

$\mathrm{ZPVE}=0.240419$

Symbol

X

Y

Z

C

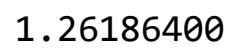

0.76181100

0.72143800

$\mathrm{H}$

0.44796900

1.29449700

1.20228300

1.23526900

$-0.66498000$

0.80129300

0.41777500

$-1.11857400$

1.35289900

$-1.71636200$

$-0.01315300$

$-0.34415300$

$-2.34558100$

$-1.21251700$

$-0.01947400$

$-2.36804200$

1.20766800

$-0.18815600$

$-3.63830600$

$-1.18196000$

0.48244100

$-1.84175800$

$-2.16099100$

$-0.15813100$

$-3.66075500$

1.22309600

0.31503200

$-1.88138800$

2.13686900

$-0.45707800$

$-4.29168100$

0.03212700

0.64950000

$-4.13562600$

$-2.10797500$

0.73790700

$-4.17525900$

2.16627700

0.44056500

$-5.30106500$

0.04970200

1.03849900

2.54543800

1.53157800

0.47157400

2.48620300

2.06739700

$-0.47636300$

2.59778600

2.30369100

1.24172200

3.79357500

0.64535200

0.51560900

4.07917400

0.47122400

1.55593400

4.62213400

1.17325600

0.04642200

3.55713700

$-0.70387700$

$-0.15573600$

3.25900200

$-0.56271400$

$-1.20198900$

4.47617400

$-1.28739200$

$-0.17794400$

2.48373200

$-1.47871000$

0.59989000

2.84776500

$-1.71679500$

1.60673700

2. 24423400

$-2.43015500$

0.12665900

Te

0.21991900

$-0.04677700$

$-1.14469600$ 


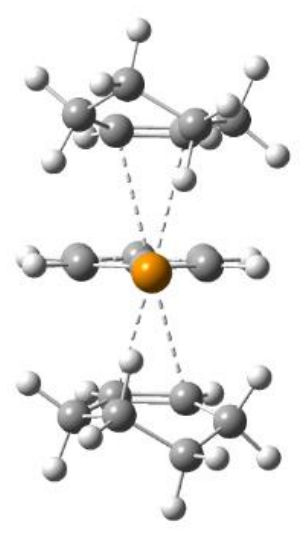

$E=-968.226665$

$H=-968.204452$

$G=-968.280288$

$\mathrm{ZPVE}=0.355136$

Symbol

$$
\text { X }
$$

Y

Z

\begin{tabular}{|c|c|c|c|}
\hline C & -1.00557300 & 2.91339800 & -0.78226900 \\
\hline C & -0.97400600 & 2.98748400 & 0.59435200 \\
\hline $\mathrm{H}$ & -1.74208300 & 2.46190400 & 1.15285000 \\
\hline $\mathrm{H}$ & -1.80889200 & 2.35397500 & -1.25159100 \\
\hline C & 1.58517000 & -1.47066000 & 0.68684500 \\
\hline $\mathrm{H}(\mathrm{IsO}=2)$ & 0.70096300 & -1.83429300 & 1.20265300 \\
\hline C & 1.61554400 & -1.51066500 & -0.66289400 \\
\hline $\mathrm{H}(\mathrm{IsO}=2)$ & 0.74288500 & -1.88004500 & -1.19408800 \\
\hline $\mathrm{C}$ & -1.44688800 & -0.19614800 & 0.00510100 \\
\hline C & -2.03277400 & -0.60963200 & -1.18870800 \\
\hline C & -2.02228600 & -0.53154600 & 1.22810700 \\
\hline C & -3.20088900 & -1.35857600 & -1.15441100 \\
\hline $\mathrm{H}$ & -1.58343100 & -0.35285200 & -2.14041400 \\
\hline C & -3.19093500 & -1.28014200 & 1.25226900 \\
\hline $\mathrm{H}$ & -1.56394000 & -0.21536200 & 2.15735300 \\
\hline C & -3.77809000 & -1.69214300 & 0.06352800 \\
\hline $\mathrm{H}$ & -3.65827600 & -1.68147000 & -2.08015300 \\
\hline $\mathrm{H}$ & -3.64066200 & -1.54176700 & 2.20083300 \\
\hline $\mathrm{H}$ & -4.68826200 & -2.27653000 & 0.08641000 \\
\hline C & 2.76582500 & -1.06359800 & 1.51958600 \\
\hline $\mathrm{H}(\mathrm{IsO}=2)$ & 2.43526300 & -0.43216700 & 2.34744100 \\
\hline $\mathrm{H}(\mathrm{IsO}=2)$ & 3.17399000 & -1.96930500 & 1.98144200 \\
\hline C & 2.84555700 & -1.19735700 & -1.46846400 \\
\hline $\mathrm{H}(\mathrm{IsO}=2)$ & 2.70527000 & -0.24819500 & -1.99792400 \\
\hline $\mathrm{H}(\mathrm{IsO}=2)$ & 2.94379600 & -1.95422800 & -2.24940900 \\
\hline C & 3.83957700 & -0.36905500 & 0.68596200 \\
\hline $\mathrm{H}(\mathrm{IsO}=2)$ & 3.51255500 & 0.64707600 & 0.43581000 \\
\hline $\mathrm{H}(\mathrm{ISO}=2)$ & 4.75327300 & -0.26547100 & 1.27022700 \\
\hline C & 4.10200700 & -1.14554000 & -0.60078900 \\
\hline $\mathrm{H}(\mathrm{ISO}=2)$ & 4.92283500 & -0.70078300 & -1.16229500 \\
\hline
\end{tabular}




$\begin{array}{lrrr}\text { H(Iso=2) } & 4.40535300 & -2.16428000 & -0.34552400 \\ \mathrm{C} & -0.12076600 & 3.98372600 & 1.33969400 \\ \mathrm{H} & -0.75536800 & 4.44797800 & 2.09761100 \\ \mathrm{H} & 0.66295000 & 3.46223400 & 1.89466900 \\ \mathrm{C} & -0.15601300 & 3.79354900 & -1.65281500 \\ \mathrm{H} & -0.81653700 & 4.57934600 & -2.03805700 \\ \mathrm{H} & 0.19207400 & 3.23744600 & -2.52375200 \\ \mathrm{C} & 0.48308900 & 5.04386800 & 0.41731600 \\ \mathrm{H} & -0.27974100 & 5.78739400 & 0.17240200 \\ \mathrm{H} & 1.28063300 & 5.57000800 & 0.93995200 \\ \mathrm{C} & 0.99712000 & 4.42264800 & -0.87766800 \\ \mathrm{H} & 1.48916300 & 5.17161200 & -1.49666900 \\ \mathrm{H} & 1.75345200 & 3.66180700 & -0.65114700 \\ \mathrm{Te} & 0.33089200 & 0.92739100 & -0.03787900\end{array}$

Transition State 6d

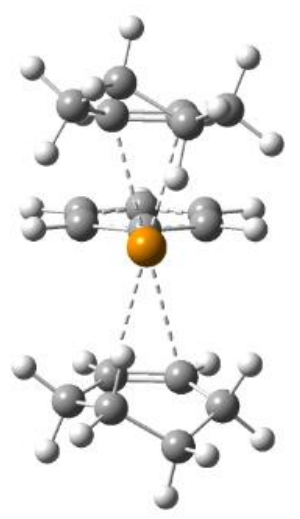

$E=-968.226728$

$H=-968.205400$

$G=-968.278357$

$\mathrm{ZPVE}=0.355036$

Imaginary Frequency $=-27.0468 \mathrm{~cm}^{-1}$

Symbol

$\mathrm{C}$
$\mathrm{C}$
$\mathrm{H}($ Iso $=2)$
$\mathrm{H}($ Iso $=2)$
$\mathrm{C}$
$\mathrm{H}$
$\mathrm{C}$
$\mathrm{H}$
$\mathrm{C}$
$\mathrm{C}$
$\mathrm{C}$
$\mathrm{C}$

X

$$
\begin{array}{r}
-1.04271800 \\
-1.01895800 \\
-1.76518700 \\
-1.82154400 \\
1.52943300 \\
0.64811900 \\
1.56785900 \\
0.70329900 \\
-1.45069600 \\
-2.04860400 \\
-2.04968500 \\
-3.24916900
\end{array}
$$

Y

$$
\begin{array}{rr}
2.97720900 & -0.80450300 \\
3.06759200 & 0.55508900 \\
2.52080100 & 1.12371200 \\
2.38389600 & -1.27426500 \\
-1.37326200 & 0.72686100 \\
-1.74809800 & 1.23877600 \\
-1.42646300 & -0.63461500 \\
-1.81800100 & -1.16219900 \\
-0.18451000 & 0.00048700 \\
-0.57951300 & -1.19383200 \\
-0.48339300 & 1.22184600 \\
-1.27541200 & -1.16186600
\end{array}
$$
$-0.80450300$
0.55508900
1.12371200
$-1.27426500$
0.72686100
1.23877600
$-0.63461500$
$-1.16219900$
$-1.19383200$
1.22184600
$-1.16186600$ 


\begin{tabular}{|c|c|c|c|}
\hline $\mathrm{H}$ & -1.58407100 & -0.34696700 & -2.14450000 \\
\hline C & -3.25051900 & -1.17924900 & 1.24408600 \\
\hline $\mathrm{H}$ & -1.58528300 & -0.17728300 & 2.15148500 \\
\hline C & -3.84810000 & -1.57398700 & 0.05464700 \\
\hline $\mathrm{H}$ & -3.71545900 & -1.58342700 & -2.08825700 \\
\hline $\mathrm{H}$ & -3.71792600 & -1.41221500 & 2.19158900 \\
\hline $\mathrm{H}$ & -4.78404900 & -2.11620000 & 0.07589200 \\
\hline C & 2.72341500 & -1.00367100 & 1.55759000 \\
\hline $\mathrm{H}$ & 2.41457800 & -0.37941300 & 2.39775000 \\
\hline $\mathrm{H}$ & 3.10698900 & -1.93132600 & 1.99756300 \\
\hline C & 2.81811900 & -1.16558700 & -1.43120700 \\
\hline $\mathrm{H}$ & 2.71032300 & -0.23246100 & -1.99258500 \\
\hline $\mathrm{H}$ & 2.90010600 & -1.95108400 & -2.18547400 \\
\hline C & 3.81180500 & -0.33160300 & 0.72579400 \\
\hline $\mathrm{H}$ & 3.50494700 & 0.68920400 & 0.46928000 \\
\hline $\mathrm{H}$ & 4.72339000 & -0.24160800 & 1.31523300 \\
\hline C & 4.06800600 & -1.12460100 & -0.55210300 \\
\hline $\mathrm{H}$ & 4.89883400 & -0.69935500 & -1.11360400 \\
\hline $\mathrm{H}$ & 4.35446100 & -2.14489500 & -0.28411400 \\
\hline C & -0.14550700 & 4.04299300 & 1.29757400 \\
\hline$H($ Iso $=2)$ & -0.75891300 & 4.51900200 & 2.06551900 \\
\hline $\mathrm{H}(\mathrm{ISO}=2)$ & 0.63377500 & 3.50049500 & 1.84161500 \\
\hline C & -0.16439600 & 3.81382400 & -1.68825100 \\
\hline $\mathrm{H}(\mathrm{IsO}=2)$ & -0.79835600 & 4.59523100 & -2.12271100 \\
\hline $\mathrm{H}(\mathrm{IsO}=2)$ & 0.19454900 & 3.21792800 & -2.52914100 \\
\hline C & 0.47266700 & 5.08964600 & 0.37075100 \\
\hline $\mathrm{H}(\mathrm{IsO}=2)$ & -0.28336100 & 5.83639600 & 0.11447900 \\
\hline $\mathrm{H}(\mathrm{ISO}=2)$ & 1.27289600 & 5.61468500 & 0.89073200 \\
\hline C & 0.98753100 & 4.44710900 & -0.91349300 \\
\hline $\mathrm{H}(\mathrm{IsO}=2)$ & 1.49224000 & 5.18224900 & -1.53907600 \\
\hline$H($ Iso $=2)$ & 1.73376200 & 3.68228600 & -0.66817200 \\
\hline $\mathrm{Te}$ & 0.36774600 & 0.87298400 & -0.04134500 \\
\hline
\end{tabular}

Dissociation Complex 6e

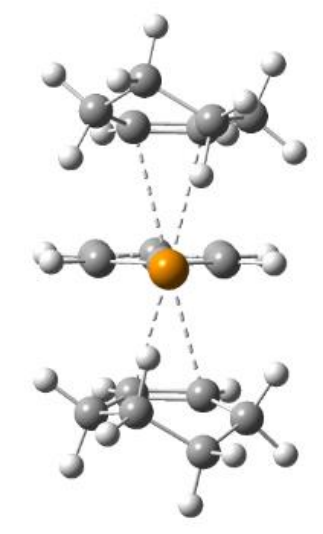

$E=-968.226823$

$H=-968.204609$

$\mathrm{G}=-968.280400$ 


\begin{tabular}{|c|c|c|c|}
\hline Symbol & $X$ & Y & Z \\
\hline C & -1.00549300 & 2.91344000 & -0.78186900 \\
\hline $\mathrm{C}$ & -0.97341300 & 2.98727600 & 0.59478300 \\
\hline $\mathrm{H}(\mathrm{ISO}=2)$ & -1.74143700 & 2.46181400 & 1.15346300 \\
\hline $\mathrm{H}(\mathrm{ISO}=2)$ & -1.80911300 & 2.35430500 & -1.25101300 \\
\hline C & 1.58508700 & -1.47107900 & 0.68622400 \\
\hline $\mathrm{H}$ & 0.70092700 & -1.83487300 & 1.20200200 \\
\hline C & 1.61544800 & -1.51088900 & $-0.6635020 e$ \\
\hline $\mathrm{H}$ & 0.74283200 & -1.88030000 & -1.19475100 \\
\hline C & -1.44719100 & -0.19596700 & 0.00523600 \\
\hline C & -2.03352700 & -0.60918600 & -1.18844100 \\
\hline C & -2.02223700 & -0.53145400 & 1.22838500 \\
\hline C & -3.20176900 & -1.35792200 & -1.15386800 \\
\hline $\mathrm{H}$ & -1.58443800 & -0.35236500 & -2.14025400 \\
\hline C & -3.19100900 & -1.27984500 & 1.25282100 \\
\hline $\mathrm{H}$ & -1.56352300 & -0.21549700 & 2.15752700 \\
\hline C & -3.77863300 & -1.69155700 & 0.06420900 \\
\hline $\mathrm{H}$ & -3.65951300 & -1.68060600 & $-2.0795080 e$ \\
\hline $\mathrm{H}$ & -3.64046600 & -1.54153800 & 2.20149400 \\
\hline $\mathrm{H}$ & -4.68890800 & -2.27577600 & 0.08730300 \\
\hline C & 2.76566300 & -1.06391200 & 1.51902500 \\
\hline $\mathrm{H}$ & 2.43497700 & -0.43257800 & 2.34690800 \\
\hline $\mathrm{H}$ & 3.17395000 & -1.96957900 & 1.98084900 \\
\hline C & 2.84539700 & -1.19721100 & -1.46902500 \\
\hline $\mathrm{H}$ & 2.70497200 & -0.24792300 & -1.99823100 \\
\hline $\mathrm{H}$ & 2.94375000 & -1.95385500 & -2.25017200 \\
\hline C & 3.83936400 & -0.36913400 & 0.68551200 \\
\hline $\mathrm{H}$ & 3.51230600 & 0.64703200 & 0.43553200 \\
\hline $\mathrm{H}$ & 4.75304200 & -0.26561400 & 1.26981600 \\
\hline C & 4.10186000 & -1.14540000 & -0.60136200 \\
\hline $\mathrm{H}$ & 4.92262800 & -0.70047500 & -1.16282200 \\
\hline $\mathrm{H}$ & 4.40531000 & -2.16415000 & -0.34626000 \\
\hline C & -0.11979600 & 3.98331600 & 1.33999000 \\
\hline $\mathrm{H}(\mathrm{ISO}=2)$ & -0.75412500 & 4.44754000 & 2.09815200 \\
\hline $\mathrm{H}(\mathrm{ISO}=2)$ & 0.66402700 & 3.46166200 & 1.89465200 \\
\hline C & -0.15607600 & 3.79360200 & -1.65254000 \\
\hline $\mathrm{H}(\mathrm{ISO}=2)$ & -0.81661800 & 4.57957200 & -2.03740800 \\
\hline $\mathrm{H}(\mathrm{IsO}=2)$ & 0.19161600 & 3.23760800 & -2.52370300 \\
\hline C & 0.48390900 & 5.04350400 & 0.41756600 \\
\hline $\mathrm{H}(\mathrm{ISO}=2)$ & -0.27888200 & 5.78719000 & 0.17301700 \\
\hline $\mathrm{H}(\mathrm{ISO}=2)$ & 1.28171400 & 5.56943700 & 0.94001000 \\
\hline C & 0.99741300 & 4.42237900 & -0.87767400 \\
\hline $\mathrm{H}(\mathrm{ISO}=2)$ & 1.48940100 & 5.17133100 & -1.49673300 \\
\hline $\mathrm{H}(\mathrm{ISO}=2)$ & 1.75367900 & 3.66136700 & -0.65149000 \\
\hline $\mathrm{Te}$ & 0.33073100 & 0.92731200 & -0.03813700 \\
\hline
\end{tabular}


Telluriranium Product Ion 6f

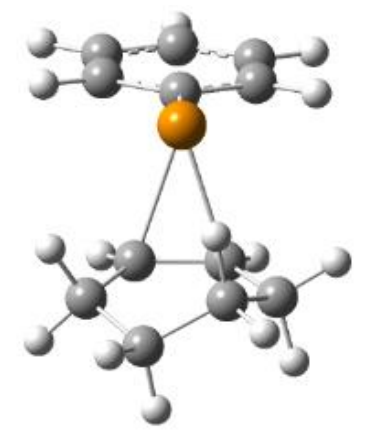

$E=-733.741793$

$H=-733.727215$

$G=-733.783777$

$\mathrm{ZPVE}=0.206750$

Symbol

X

Y

Z

\section{C}

$\mathrm{H}(\mathrm{ISO}=2)$

$\mathrm{C}$

$\mathrm{H}(\mathrm{IsO}=2)$

C

C

C

$\mathrm{C}$

$\mathrm{H}$

C

$\mathrm{H}$

C

$\mathrm{H}$

$\mathrm{H}$

$\mathrm{H}$

C

$\mathrm{H}(\mathrm{ISO}=2)$

$\mathrm{H}(\mathrm{ISO}=2)$

$\mathrm{C}$

$\mathrm{H}(\mathrm{ISO}=2)$

$\mathrm{H}(\mathrm{ISO}=2)$

$\mathrm{C}$

$\mathrm{H}(\mathrm{ISO}=2)$

$\mathrm{H}(\mathrm{ISO}=2)$

C

$\mathrm{H}(\mathrm{ISO}=2)$

$\mathrm{H}(\mathrm{ISO}=2)$

Te

0.76162900

1.29434400

$-0.66510400$

$-1.11884000$

$-0.01318900$

$-1.21246200$

1.20768200

$-1.18177100$

$-2.16096700$

1.22324600

2.13681400

0.03236200

$-2.10772000$

2.16646500

0.05004200

1.53148100

2.06770400

2.30331800

0.64534200

0.47122500

1.17331900

$-0.70390000$

$-0.56280500$

$-1.28739400$

$-1.47875300$

$-1.71683300$

$-2.43023200$

$-0.04703200$
0.72227400

1.20318700

0.80216200

1.35398000

$-0.34369000$

$-0.01895800$

$-0.18828700$

0.48229800

$-0.15708900$

0.31424100

$-0.45716800$

0.64869000

0.73778700

0.43928200

1.03717100

0.47196500

$-0.47571100$

1.24238100

0.51501600

1.55512700

0.04524600

$-0.15615200$

$-1.20225500$

$-0.17889000$

0.60008100

1.60673000

0.12702300

$-1.14375600$ 
Transition State $2 \mathrm{~g}$

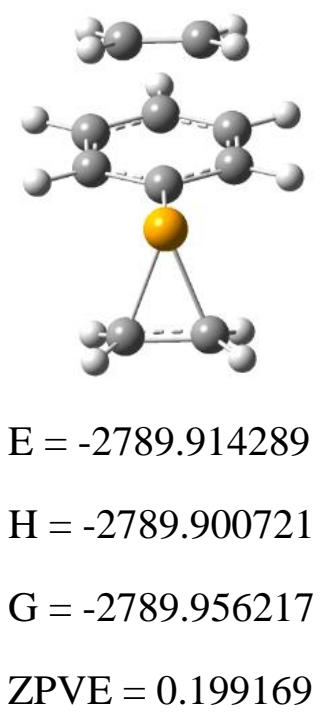

Imaginary Frequency $=-31.9658 \mathrm{~cm}^{-1}$

$\begin{array}{lrrr}\text { C } & 1.35364300 & -2.35744600 & 0.72252300 \\ \mathrm{C} & 1.35377100 & -2.35750000 & -0.72220800 \\ \mathrm{H} & 0.43780800 & -2.57315100 & -1.25547300 \\ \mathrm{H} & 0.43758600 & -2.57305700 & 1.25564200 \\ \mathrm{H} & 2.27989600 & -2.52640000 & 1.25547100 \\ \mathrm{H} & 2.28011800 & -2.52649400 & -1.25497900 \\ \mathrm{C} & 1.06160700 & 2.80974200 & -0.66284800 \\ \mathrm{H} & 1.97669800 & 2.92814400 & -1.23064900 \\ \mathrm{H} & 0.13989700 & 2.73030700 & -1.22777200 \\ \mathrm{C} & 1.06154200 & 2.80969400 & 0.66307200 \\ \mathrm{H} & 0.13977600 & 2.73021400 & 1.22789700 \\ \mathrm{H} & 1.97657600 & 2.92805700 & 1.23097200 \\ \mathrm{C} & -0.59607600 & -0.23323300 & -0.00008300 \\ \mathrm{C} & -1.25504400 & -0.10796000 & 1.21524900 \\ \mathrm{C} & -1.25483900 & -0.10803900 & -1.21553500 \\ \mathrm{C} & -2.62234500 & 0.12774200 & 1.20578200 \\ \mathrm{H} & -0.71923800 & -0.18666500 & 2.15305700 \\ \mathrm{C} & -2.62214200 & 0.12766400 & -1.20631300 \\ \mathrm{H} & -0.71887400 & -0.18680600 & -2.15324800 \\ \mathrm{C} & -3.30129900 & 0.24359600 & -0.00032700 \\ \mathrm{H} & -3.15436800 & 0.22337900 & 2.14253000 \\ \mathrm{H} & -3.15400700 & 0.22324200 & -2.14315700 \\ \mathrm{H} & -4.36694800 & 0.42899600 & -0.00042300 \\ \mathrm{Se} & 1.30861800 & -0.47692800 & 0.00008700\end{array}$

